\title{
Géométrie réelle des dessins d'enfant : une étude des composantes irréductibles
}

\author{
par LAYLA PHARAMOND DIT D'COSTA ${ }^{1}$
}

\begin{abstract}
RÉSUMÉ. Dans cet article nous nous intéressons aux propriétés des composantes irréductibles associées à la géométrie réelle d'un dessin d'enfant. Plus précisément, nous étudions les composantes irréductibles de la courbe $\Gamma$ dont l'ensemble des points réels est l'image réciproque de $\mathbf{P}^{1}(\mathbf{R})$ par une fonction de Belyi d'un dessin d'enfant.
\end{abstract}

ABSTRACT. In this paper, are studied the properties of the irreducibles components associated with the real geometry of a dessin d'enfant. In other words, we give a description of the irreducible components of the curve $\Gamma$, the real points of which correspond to the preimage of the real projective line by a Belyi funcion of a dessin d'enfant.

Dans tout ce texte, $\mathrm{X}$ désigne une courbe algébrique, projective et lisse sur $\mathbf{C}, f$ un morphisme fini (de $\mathbf{C}$-schémas) de $\mathrm{X}$ dans $\mathbf{P}_{\mathbf{C}}^{1}$, non ramifié au-dessus de $\mathbf{P}_{\mathbf{C}}^{1}-\{0,1, \infty\}$, et $n$ le degré de $f$.

On note $f$ l'application de $\mathrm{X}(\mathbf{C})$ dans $\mathbf{P}_{\mathbf{C}}^{1}(\mathbf{C})$ qui se déduit de la précédente par passage aux points complexes : c'est un revêtement topologique ramifié fini de $\mathbf{P}_{\mathbf{C}}^{1}(\mathbf{C})$, non ramifié au dessus de $\mathbf{P}_{\mathbf{C}}^{1}(\mathbf{C})-\{0,1, \infty\}([2]$, $\S$ B.4.1).

On associe à ce revêtement un dessin d'enfant D ([2], §B.3.3) tracé sur la surface topologique $\mathrm{X}(\mathbf{C})$, qui possède une triangulation adaptée canonique $\mathrm{T}([2], \S \mathrm{B} .5 .1$, exemple). La réunion des sommets et des arêtes de la triangulation $\mathrm{T}$ est l'image réciproque par $\underline{f}$ de $\mathbf{P}_{\mathbf{C}}^{1}(\mathbf{R})$ : c'est un sous-ensemble algébrique réel de $\mathrm{X}(\mathbf{C})$.

On note $\Gamma$ la courbe définie sur $\mathbf{R}$, dont $\underline{f}^{-1}\left(\mathbf{P}_{\mathbf{C}}^{1}(\mathbf{R})\right)$ est l'ensemble des points réels $([2], \S 1.1)$ et $g: \Gamma \rightarrow \mathbf{P}_{\mathbf{R}}^{1}$ le morphisme correspondant.

$\mathrm{Au} \S 1$, nous précisons $\Gamma$ et rappelons quelques unes de ses propriétés générales. La géométrie réelle du dessin d'enfant est l'ensemble des propriétés de $\Gamma$.

Manuscrit reçu le ler février 2004.

1. Cet article n'aurait pas vu le jour sans les multiples contributions de Joseph Oesterlé. Je l'en remercie châleureusement. 
Notons $\left(\mathrm{E}, \sigma_{0}, \sigma_{1}\right)$ le dessin combinatoire associé à $\mathrm{D}([2], \S \mathrm{B} .2 .2)$ : E est l'ensemble des arêtes de $\mathrm{D}$, i.e. l'ensemble des composantes connexes de $f^{-1}(] 0,1[)$; si $a$ est une telle arête, les arêtes ayant même extrémité de type 0 (resp. de type 1) que $a$ sont munies d'un ordre cyclique déduit de l'orientation de la surface $\mathrm{X}(\mathbf{C})$ en ce point, et $\sigma_{0}(a)$ (resp. $\sigma_{1}(a)$ ) est le successeur de $a$ pour cet ordre cyclique.

Par définition, l'ensemble $E$ paramètre l'ensemble des arêtes de de type $\{0,1\}$ de la triangulation $\mathrm{T}$. Nous indiquons au $\S 2$, de quelle manière on peut également paramétrer l'ensemble des arêtes de type $\{\infty, 0\}$ (resp. de type $\{1, \infty\})$ de $\mathrm{T}$ par $\mathrm{E}$.

Notons $\underline{g}$ l'application continue de $\Gamma(\mathrm{C})$ dans $\mathbf{P}^{1}(\mathbf{C})$ déduite du morphisme $g_{(\mathbf{C})}: \Gamma_{(\mathbf{C})} \rightarrow \mathbf{P}^{1}$. Nous décrivons au $\S 3$ une paramétrisation par $\mathrm{E} \times \mathrm{E}$ des composantes connexes de $\underline{g}^{-1}(] 0,1[), \underline{g}^{-1}(] 1, \infty[)$ et $\underline{g}^{-1}(] \infty, 0[)$. Nous la comparons à celle du $\S 2$, pour les composantes contenues dans $\Gamma(\mathrm{R})$.

$\mathrm{Au} \S 4$, nous indiquons comment cette paramétrisation permet de déterminer, à partir du dessin combinatoire $\left(\mathrm{E}, \sigma_{0}, \sigma_{1}\right)$, la liste des composantes connexes de $\widetilde{\Gamma}(\mathbf{R})$ : il s'agit de décrire la manière dont les arêtes de $\mathrm{T}$ se succèdent sur une telle composante connexe, ce que nous faisons en utilisant la subdivision barycentrique de $\mathrm{T}$.

Ces résultats et ceux de [2], $\S 2$, nous permettent, au $\S 5$, de déterminer le nombre de composantes connexes de l'ensemble des points réels de chaque composante irréductible de $\widetilde{\Gamma}$; ce nombre peut être $\geq 2$, comme le montre un exemple donné au $\S 6$.

Enfin au $\$ 7$, après des rappels sur les diviseurs, le groupe de Picard et ses propriétés de fonctorialité, nous étudions les diviseurs de $\mathrm{X} \times \mathrm{X}^{*}$ définis par les composantes irréductibles de $\Gamma_{(\mathbf{C})}$ Nous notons [C] la classe d'une telle composante dans le groupe de $\mathrm{Picard} \operatorname{Pic}\left(\mathrm{X} \times \mathrm{X}^{*}\right)$. Nous considérons plus particulièrement le cas où la courbe $\mathrm{X}$ est connexe et de genre 0 , i.e. isomorphe à $\mathbf{P}^{1}$. Dans ce cas $\operatorname{Pic}\left(\mathrm{X} \times \mathrm{X}^{*}\right)$ s'identifie à $\mathbf{Z} \times \mathbf{Z}$ et $[\mathrm{C}]$ à un couple d'entiers appelé le bidegré de $\mathrm{C}$; nous l'exprimons en termes de l'orbite $\mathcal{O}$ de $\left\langle\Sigma_{0}, \Sigma_{1}\right\rangle$ dans $\mathrm{E} \times \mathrm{E}$ associée à $\mathrm{C}$. Le choix d'un isomorphisme $\mathrm{X} \approx \mathbf{P}^{1}$ permet de considérer la trace de $\mathrm{C}$ sur le plan affine $\mathbf{A}^{1} \times \mathbf{A}^{1}$. C'est une courbe affine dont nous calculons le degré; il dépend du point de $\mathrm{X}$ qui correspond au point à l'infini de $\mathbf{P}^{1}$ par l'isomorphisme choisi. Nous considérons ensuite le cas où $\mathrm{X}$ est connexe de genre quelconque. Auquel cas, la structure du groupe de Picard de $\mathrm{X} \times \mathrm{X}^{*}$ est plus compliquée : on dispose d'une suite exacte canonique

$$
0 \rightarrow \operatorname{Pic}(\mathrm{X}) \times \operatorname{Pic}\left(\mathrm{X}^{*}\right) \rightarrow \operatorname{Pic}\left(\mathrm{X} \times \mathrm{X}^{*}\right) \rightarrow \operatorname{Hom}\left(\operatorname{Jac}(\mathrm{X}), \operatorname{Jac}\left(\mathrm{X}^{*}\right)\right) \rightarrow 0,
$$

où $\operatorname{Jac}(\mathrm{X})$ désigne la variété Jacobienne de $\mathrm{X}$; les flèches sont précisées au $\S 7.1 .6$. Nous montrons par un exemple que les images des classes [C] des 
composantes irréductibles $\mathrm{C}$ de $\Gamma_{(\mathbf{C})}$ dans $\operatorname{Hom}\left(\operatorname{Jac}(\mathrm{X}), \operatorname{Jac}\left(\mathrm{X}^{*}\right)\right)$ peuvent être non nulles bien que leur somme soit 0 .

\section{La courbe $\Gamma$ associée à un dessin d'enfant}

La courbe $\Gamma$ (celle qui est telle que $f^{-1}\left(\mathbf{P}_{\mathbf{C}}^{1}(\mathbf{R})\right)$ est l'ensemble des points réels $([2], \S 1.1))$ peut se définir comme le produit fibré $\mathbf{P}_{\mathbf{R}}^{1} \times \prod_{\mathbf{C} / \mathbf{R}} \mathbf{P}_{\mathbf{C}}^{1} \prod_{\mathbf{C} / \mathbf{R}} \mathrm{X}$ :

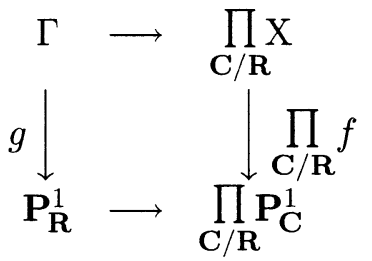

où $\prod_{\mathbf{C} / \mathbf{R}} \mathrm{X}, \prod_{\mathbf{C} / \mathbf{R}} \mathbf{P}_{\mathbf{C}}^{1}$, sont les restrictions de Weil à $\mathbf{R}$ de $\mathrm{X}$ et $\mathbf{P}_{\mathbf{C}}^{1}$ respectivement et $\prod_{\mathbf{C} / \mathbf{R}} f: \prod_{\mathbf{C} / \mathbf{R}} \mathrm{X} \rightarrow \prod_{\mathbf{C} / \mathbf{R}} \mathbf{P}_{\mathbf{C}}^{1}$ le morphisme de $\mathbf{R}$-schémas déduit de $f$ par restriction de Weil. Le morphisme $g: \Gamma \rightarrow \mathbf{P}_{\mathbf{R}}^{1}$ est défini par restriction de $\prod_{\mathbf{C} / \mathbf{R}} f$ à $\Gamma$ et correspond donc à la projection sur le premier facteur de $\mathbf{P}_{\mathbf{R}}^{1} \times \prod_{\mathbf{C} / \mathbf{R}} \mathbf{P}_{\mathbf{C}}^{1} \prod_{\mathbf{C} / \mathbf{R}} \mathbf{X} \rightarrow \mathbf{P}_{\mathbf{R}}^{1}$ Notons $\mathrm{X}^{*}$ la courbe algébrique complexe conjuguée de $\mathrm{X}([2], \S \mathrm{A} .1 .3)$ et $\Delta: \mathbf{P}_{\mathbf{C}}^{1} \rightarrow \mathbf{P}_{\mathbf{C}}^{1} \times \mathbf{P}_{\mathbf{C}}^{1}$ le morphisme diagonale. Le carré cartésien déduit du précédent par passage au complexifié s'identifie $([2], \S 1.2)$ à

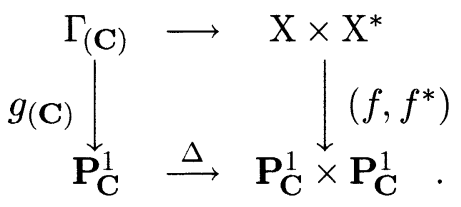

Notons $\widetilde{\Gamma}$ la normalisée de $\Gamma$, et $\widetilde{g}$ le morphisme composé $\widetilde{\Gamma} \longrightarrow \Gamma \stackrel{g}{\longrightarrow} \mathbf{P}_{\mathbf{R}}^{1}$. Nous noterons $\widetilde{\Gamma}_{(\mathbf{C})}$ et $\widetilde{g}_{(\mathbf{C})}$ le revêtement ramifié et le morphisme $\widetilde{\Gamma}_{(\mathbf{C})} \rightarrow$ $\mathbf{P}_{\mathbf{C}}^{1}$ déduits respectivement de $\widetilde{\Gamma}$ et de $\widetilde{g}$ par passage au complexifié. Notons $\left(\mathrm{E}, \sigma_{0}, \sigma_{1}\right)$ le dessin combinatoire associé à $\mathrm{D}([2], \S \mathrm{B} .2 .2)$ : E est l'ensemble des arêtes de $\mathrm{D}$, i.e. l'ensemble des composantes connexes de $\underline{f}^{-1}(] 0,1[)$; si $a$ est une telle arête, les arêtes ayant même extrémité de type 0 (resp. de type 1) que $a$ sont munies d'un ordre cyclique déduit de l'orientation de la surface $\mathrm{X}(\mathbf{C})$ en ce point, et $\sigma_{0}(a)$ (resp. $\sigma_{1}(a)$ ) est le successeur de $a$ pour cet ordre cyclique. On associe de manière analogue un dessin d'enfant et un dessin combinatoire au revêtement ramifié $\widetilde{g}_{(\mathbf{C})}: \widetilde{\Gamma}_{(\mathbf{C})} \rightarrow \mathbf{P}_{\mathbf{C}}^{1}$. Ce dernier est canoniquement isomorphe à $\left(\mathrm{E} \times \mathrm{E}, \Sigma_{0}, \Sigma_{1}\right)$, où $\Sigma_{0}=\sigma_{0} \times \sigma_{0}^{-1}$ et $\Sigma_{1}=\sigma_{1} \times \sigma_{1}^{-1}([2], \S 1.5$, prop. 1.2$)$. Nous notons $\Gamma(\mathbf{R})$ et $\widetilde{\Gamma}(\mathbf{R})$ les 
ensembles de points réels de $\Gamma$ et de $\widetilde{\Gamma}$, munis de leurs topologies naturelles d'espaces analytiques réels. Rappelons que $\Gamma(\mathbf{R})$ s'identifie canoniquement à l'image réciproque de $\mathbf{P}^{1}(\mathbf{R})$ par l'application $f: \mathrm{X}(\mathbf{C}) \rightarrow \mathbf{P}^{1}(\mathbf{C})$, i.e. au 1-squelette de la triangulation canonique $\mathrm{T}$ de $\mathrm{X}(\mathbf{C})$ associée au revêtement ramifié $(\mathrm{X}, f)$. Par ailleurs $\widetilde{\Gamma}(\mathbf{R})$ est une courbe analytique réelle (lisse) compacte; toutes ses composantes connexes sont donc isomorphes (en tant que variétés analytiques réelles) à des cercles.

\section{Paramétrisation des arêtes de la triangulation canonique $T$ de $\mathrm{X}(\mathrm{C})$}

Comme $\left(\mathrm{E}, \sigma_{0}, \sigma_{1}\right)$ le dessin combinatoire associé au revêtement ramifié $(\mathrm{X}, f), \mathrm{E}$ est l'ensemble des arêtes de de type $\{0,1\}$ de la triangulation $\mathrm{T}$, i.e. l'ensemble des composantes connexes de $\underline{f}^{-1}(] 0,1[)$.

Chaque arête de $\mathrm{T}$ est arête de deux faces exactement, l'une positive et l'autre négative; chaque face de $\mathrm{T}$ a trois arêtes exactement, de types $\{0,1\}$, $\{\infty, 0\}$ et $\{1, \infty\}$ respectivement. On peut paramétrer l'ensemble des faces positives (resp. des faces négatives) de $\mathrm{T}$ par $\mathrm{E}$ en associant à chaque face son unique arête de type $\{0,1\}$.

Il y a plusieurs manières de paramétrer les arêtes de type $\{\infty, 0\}$ (resp. de type $\{1, \infty\}$ ) de $\mathrm{T}$ par E. Nous en considérons deux, que nous appelons respectivement la paramétrisation positive et la paramétrisation négative. La paramétrisation positive associe à une arête $b$ de type $\{\infty, 0\}$ (resp. de type $\{1, \infty\}$ ) l'arête de type $\{0,1\}$ de l'unique face positive dont $b$ est aussi une arête. La paramétrisation négative se définit de manière analogue à partir des faces négatives.

La figure ci-dessous montre comment les deux paramétrisations s'articulent autour d'une même arête $a$ de type $\{0,1\}$.

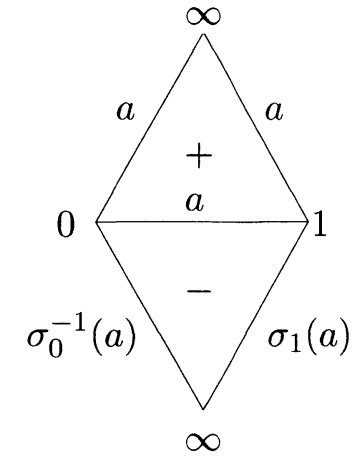

Paramétrisation positive

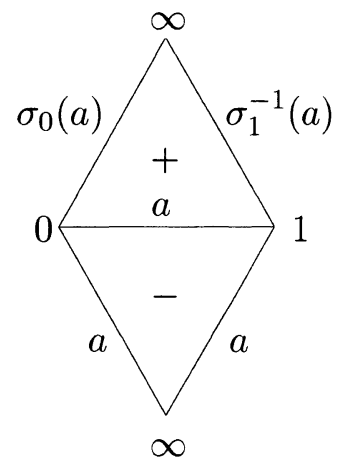

Paramétrisation négative 
Il existe une paramétrisation canonique des sommets de $\mathrm{T}$ de type 0,1 et $\infty \operatorname{par}\left\langle\sigma_{0}\right\rangle \backslash \mathrm{E},\left\langle\sigma_{1}\right\rangle \backslash \mathrm{E}$ et $\left\langle\sigma_{\infty}\right\rangle \backslash \mathrm{E}$ respectivement ([2], §1.6). Soient $i, j$ deux éléments distincts de $\{0,1, \infty\}$. Lorsqu'on paramètre positivement les arêtes, l'application qui à une arête de type $\{i, j\}$ de $\mathrm{T}$ associe son extrémité de type $i$ s'interprète comme la surjection canonique $\mathrm{E} \rightarrow\left\langle\sigma_{i}\right\rangle \backslash \mathrm{E}$. (Cela n'est pas vrai pour la paramétrisation négative, lorsque $i=\infty$.)

Sauf mention du contraire, nous utiliserons désormais toujours la paramétrisation positive des arêtes de $\mathrm{T}$.

\section{Paramétrisation des arêtes de $\Gamma(\mathbf{C})$ ou $\widetilde{\Gamma}(\mathbf{C})$}

\subsection{Les triangulations canoniques de $\Gamma(\mathbf{C})$ et $\widetilde{\Gamma}(\mathbf{C})$.}

Rappelons que $\underline{g}: \Gamma(\mathrm{C}) \rightarrow \mathbf{P}^{1}(\mathbf{C})$ désigne l'application continue déduite du morphisme $g_{(\mathbf{C})}: \Gamma_{(\mathbf{C})} \rightarrow \mathbf{P}^{1}(\mathbf{C})$. L'image réciproque par $\underline{g}$ de la triangulation canonique de $\mathbf{P}^{1}(\mathbf{R})$ ([2], B.3.3, remarque 2) définit une triangulation, dite canonique, de $\Gamma(\mathrm{C})$, à sommets tricoloriés. (La notion de triangulation utilisée ici est la même que dans [2], §B.5.1, à ceci près que $\Gamma(\mathrm{C})$ n'est pas une surface topologique au voisinage des points au-dessus de 0,1 et $\infty$ ).

Les sommets, arêtes et faces de cette triangulation seront simplement appelés sommets, arêtes et faces de $\Gamma(\mathbf{C})$. Les sommets de type $0,1, \infty$ de $\Gamma(\mathbf{C})$ sont donc les points de $\underline{g}^{-1}(0), \underline{g}^{-1}(1), \underline{g}^{-1}(\infty)$, les arêtes de type $\{\infty, 0\},\{0,1\},\{1, \infty\}$ sont les composantes connexes de $\underline{g}^{-1}(] \infty, 0[)$, $\underline{g}^{-1}(] 0,1[), \underline{g}^{-1}(] 1, \infty[)$, et les faces positives et négatives les composantes connexes de $\underline{g}^{-1}\left(\mathfrak{H}_{+}\right)$et $\underline{g}^{-1}\left(\mathfrak{H}_{-}\right)$, avec $\mathfrak{H}_{+}\left(\right.$resp. $\left.\mathfrak{H}_{-}\right)$le demi-plan supérieur (resp. inférieur) de Poincaré.

On définit de manière analogue la triangulation canonique de $\widetilde{\Gamma}(\mathrm{C})$. Ce n'est autre que la triangulation canonique associée au revêtement ramifié $\widetilde{g}_{(\mathbf{C})}: \widetilde{\Gamma}_{(\mathbf{C})} \rightarrow \mathbf{P}^{1}([2]$. B.3.3). Les arêtes de type $\{\infty, 0\},\{0,1\},\{1, \infty\}$, les faces positives et les faces négatives de $\widetilde{\Gamma}(\mathrm{C})$ sont en bijection canonique avec celles de $\Gamma(\mathrm{C})$; il n'en est pas de même des sommets.

\subsection{Paramétrisation des arêtes de $\Gamma(\mathbf{C})$ et $\widetilde{\Gamma}(\mathbf{C})$.}

$\mathrm{Si}\left(\mathrm{E}, \sigma_{0}, \sigma_{1}\right)$ est le dessin combinatoire associé au revêtement ramifié $(\mathrm{X}, f)$, celui associé au revêtement ramifié conjugué $\left(\mathrm{X}^{*}, f^{*}\right)$ s'identifie à $\left(\mathrm{E}, \sigma_{0}^{-1}, \sigma_{1}^{-1}\right)$, et celui associé au revêtement ramifié $\left(\widetilde{\Gamma}_{(\mathbf{C})}, \widetilde{g}_{(\mathbf{C})}\right)$ à $(\mathrm{E} \times$ $\left.\mathrm{E}, \Sigma_{0}, \Sigma_{1}\right)$, où $\Sigma_{0}=\sigma_{0} \times \sigma_{0}^{-1}$ et $\Sigma_{1}=\sigma_{1} \times \sigma_{1}^{-1}([2], \S 1.5$, prop. 1.2).

Soit $\mathrm{T}^{*}$ la triangulation canonique de $\mathrm{X}^{*}(\mathbf{C})$ associée au revêtement $\left(\mathrm{X}^{*}, f^{*}\right)$. Soient $i, j$ deux éléments distincts de $\{0,1, \infty)$. Lorsqu'on plonge $\Gamma(\mathbf{C})$ dans $\mathrm{X}(\mathbf{C}) \times \mathrm{X}^{*}(\mathbf{C})$, toute arête de $\Gamma(\mathbf{C})$ de type $\{i, j\}$ est le produit fibré (au-dessus de $\mathbf{P}^{1}(\mathbf{C})$ ) d'une arête de $\mathrm{T}$ et d'une arête de $\mathrm{T}^{*}$, toutes deux de type $\{i, j\}$. Le choix des paramétrisations positives des 
arêtes de type $\{i, j\}$ de $\mathrm{T}$ par $\mathrm{E}$ et de $\mathrm{T}^{*}$ par $\mathrm{E}$ définit donc une paramétrisation des arêtes de type $\{i, j\}$ de $\Gamma(\mathbf{C})$ par $\mathrm{E} \times \mathrm{E}$, et par suite aussi une paramétrisation des arêtes de type $\{i, j\}$ de $\widetilde{\Gamma}(\mathbf{C})$ par $\mathrm{E} \times \mathrm{E}$. Cette dernière n'est autre que la paramétrisation positive, au sens du $\S 2$, associée au revêtement ramifié $\left(\widetilde{\Gamma}_{(\mathbf{C})}, \widetilde{g}_{(\mathbf{C})}\right)$.

Sauf mention du contraire, c'est toujours de cette manière que nous paramétrerons les arêtes de de $\Gamma(\mathbf{C})$ et de $\widetilde{\Gamma}(\mathbf{C})$ désormais.

3.3. Liens entre arêtes et sommets de $\Gamma(\mathbf{C})$ et de $\widetilde{\Gamma}(\mathbf{C})$.

Rappelons que les ensembles de sommets de type $0,1, \infty$ de $\Gamma(\mathbf{C})$ sont paramétrés respectivement par $\left\langle\sigma_{0}\right\rangle \backslash \mathrm{E} \times\left\langle\sigma_{0}^{-1}\right\rangle \backslash \mathrm{E},\left\langle\sigma_{1}\right\rangle \backslash \mathrm{E} \times\left\langle\sigma_{1}^{-1}\right\rangle \backslash \mathrm{E}$ et $\left\langle\sigma_{\infty}\right\rangle \backslash \mathrm{E} \times\left\langle\sigma_{\infty}^{*}\right\rangle \backslash \mathrm{E}$, où $\sigma_{\infty}=\sigma_{0}^{-1} \sigma_{1}^{-1}$ et $\sigma_{\infty}^{*}=\sigma_{0} \sigma_{1}([2], \S 1.6) ;$ les ensembles de sommets de type $0,1, \infty$ de $\widetilde{\Gamma}(\mathbf{C})$ sont paramétrés respectivement par $\left\langle\Sigma_{0}\right\rangle \backslash(\mathrm{E} \times \mathrm{E}),\left\langle\Sigma_{1}\right\rangle \backslash(\mathrm{E} \times \mathrm{E})$ et $\left\langle\Sigma_{\infty}\right\rangle \backslash(\mathrm{E} \times \mathrm{E})$, où $\Sigma_{\infty}=\sigma_{\infty} \times \sigma_{\infty}^{*}$.

Soient $i, j$ deux éléments distincts de $\{0,1, \infty\}$. Il résulte du dernier alinéa du $\S 2$ (appliqué au revêtement ramifié $\left.\left(\widetilde{\Gamma}_{(\mathbf{C})}, \widetilde{g}_{(\mathbf{C})}\right)\right)$ que l'application qui à une arête de type $\{i, j\}$ de $\widetilde{\Gamma}(\mathbf{C})$ associe son extrémité de type $i$ s'interprète, via les paramétrisations des sommets précédentes et des arêtes définies en 3.2 , comme la surjection canonique $\mathrm{E} \times \mathrm{E} \rightarrow\left\langle\Sigma_{i}\right\rangle \backslash(\mathrm{E} \times \mathrm{E})$.

On en déduit que l'application qui à une arête de type $\{i, j\}$ de $\Gamma(\mathbf{C})$ associe son extrémité de type $i$ s'interprète comme la surjection canonique de $\mathrm{E} \times \mathrm{E} \operatorname{sur}\left\langle\sigma_{i}\right\rangle \backslash \mathrm{E} \times\left\langle\sigma_{i}^{-1}\right\rangle \backslash \mathrm{E}$ si $i=0$ ou $1, \operatorname{sur}\left\langle\sigma_{\infty}\right\rangle \backslash \mathrm{E} \times\left\langle\sigma_{\infty}^{*}\right\rangle \backslash \mathrm{E}$ si $i=\infty$.

\subsection{Arêtes de $\Gamma(C)$ situées sur une composante irréductible don- née. \\ Chaque arête de $\Gamma(\mathbf{C})$ est contenue dans l'ensemble des points complexes d'une unique composante irréductible de $\Gamma_{(\mathbf{C})}$ (et ne rencontre pas les autres composantes irréductibles). Plus précisément :}

Proposition 3.1. L'arête de $\Gamma(\mathbf{C})$ de type $\{\infty, 0\}$ (resp. $\{0,1\}$; resp. $\{1, \infty\})$ paramétrée par un couple $(a, b) \in \mathrm{E} \times \mathrm{E}$ (cf. 3.2) est contenue dans l'ensemble des points complexes de la composante irréductible de $\Gamma_{(\mathbf{C})}$ paramétrée par l'orbite $\left\langle\Sigma_{0}, \Sigma_{1}\right\rangle(a, b)([2], \S 2.1 .1)$.

Les arêtes de type $\{\infty, 0\},\{0,1\}$ et $\{1, \infty\}$ paramétrées par un même couple $(a, b)$ sont contenues dans l'ensemble des points complexes d'une même composante irréductible de $\Gamma_{(\mathbf{C})}$ : en effet leurs relèvements dans $\widetilde{\Gamma}(\mathbf{C})$ sont les arêtes d'une même face de la triangulation canonique de $\widetilde{\Gamma}(\mathbf{C})$, donc sont contenues dans l'ensemble des points complexes d'une même composante connexe, i.e. irréductible, de $\widetilde{\Gamma}_{(\mathbf{C})}$.

Il suffit donc de démontrer la prop. 3.1 pour les arêtes de type $\{0,1\}$; or, dans ce cas, elle résulte des définitions mêmes des paramétrisations considérées. 


\subsection{Action de la conjugaison complexe sur les arêtes de $\Gamma(\mathbf{C})$.}

Comme $\Gamma$ est une courbe algébrique réelle, la conjugaison complexe opère sur $\Gamma(\mathbf{C})$; lorsque l'on plonge $\Gamma(\mathbf{C})$ dans $\mathrm{X}(\mathbf{C}) \times \mathrm{X}^{*}(\mathbf{C})$, cette opération s'écrit $\left(x, y^{*}\right) \mapsto\left(y, x^{*}\right)$ (où $x \mapsto x^{*}$ est l'application canonique de $\mathrm{X}(\mathbf{C})$ dans $\mathrm{X}^{*}(\mathbf{C})$ déduite de la conjugaison complexe).

L'application $x \mapsto x^{*}$ transforme la triangulation $\mathrm{T}$ de $\mathrm{X}(\mathbf{C})$ en la triangulation $\mathrm{T}^{*}$ de $\mathrm{X}^{*}(\mathbf{C})$, mais applique les faces positives de $\mathrm{T}$ sur les faces négatives de $\mathrm{T}^{*}$. Elle transforme une arête de $\mathrm{T}$ de type $\{\infty, 0\}$ (resp. $\{0,1\}$; resp. $\{1, \infty\})$ paramétrée positivement par un élément $a$ de $E$ en l'arête de $\mathrm{T}^{*}$ de même type paramétrée négativement par $a$, c'est-à-dire paramétrée positivement par $\sigma_{0}(a)$ (resp. $a$; resp. $\sigma_{1}^{-1}(a)$ ) (voir $\S 2$, figure 1 ).

L'action de la conjugaison complexe sur les arêtes de $\Gamma(\mathbf{C})$ (ou de $\widetilde{\Gamma}(\mathbf{C})$ ) s'interprète donc, via les paramétrisation de 3.2 , comme suit :

- sur les arêtes de type $\{\infty, 0\}$, par $(a, b) \mapsto\left(\sigma_{0}^{-1}(b), \sigma_{0}(a)\right)$;

- sur les arêtes de type $\{0,1\}$, par $(a, b) \mapsto(b, a)$;

- sur les arêtes de type $\{1, \infty\}$, par $(a, b) \mapsto\left(\sigma_{1}(b), \sigma_{1}^{-1}(a)\right)$.

\subsection{Arêtes réelles de $\Gamma(\mathbf{C})$.}

Pour qu'une arête de $\Gamma(\mathbf{C})$ de type $\{\infty, 0\}$ (resp. $\{0,1\} ;$ resp. $\{1, \infty\}$ ) paramétrée par un couple $(a, b) \in \mathrm{E} \times \mathrm{E}$ soit réelle, i.e. contenue dans $\Gamma(\mathbf{R})$, il faut et il suffit d'après 3.5 que l'on ait $b=\sigma_{0}(a)$ (resp. $b=a$; resp. $\left.b=\sigma_{1}^{-1}(a)\right)$.

Comme $\Gamma(\mathbf{R})$ s'identifie au 1-squelette de la triangulation canonique $\mathrm{T}$ de $\mathrm{X}(\mathbf{C})$, les arêtes réelles de $\Gamma(\mathbf{C})$ s'identifient aux arêtes de T. Le lien entre les paramétrisations considérées aux $\S 2$ et 3.2 est le suivant : à l'arête de $T$ de type $\{\infty, 0\}$ (resp. $\{0,1\}$; resp. $\{1, \infty\}$ ) paramétrée par un élément $a \in \mathrm{E}$ correspond l'arête réelle de $\Gamma(\mathbf{C})$ de même type paramétrée par $\left(a, \sigma_{0}(a)\right)$ (resp. $\left.(a, a) ; \operatorname{resp} .\left(a, \sigma_{1}^{-1}(a)\right)\right)$.

\subsection{Interprétation en termes de dessins triangulés.}

Soit $\mathrm{D}$ un dessin d'enfant représentant le revêtement ramifié $(\mathrm{X}, f)$; ses arêtes sont paramétrées par E. Pour représenter la triangulation $\mathrm{T}$, on choisit une triangulation adaptée $\mathcal{T}$ de $\mathrm{D}([2], \S B .5 .1)$. Les arêtes de $\mathcal{T}$ correspondent donc bijectivement aux arêtes de $\mathrm{T}$, i.e. aux arêtes réelles de $\Gamma(\mathbf{C})$; les paramétrisations de 3.2 définissent un étiquetage de ces arêtes par des éléments de $\mathrm{E} \times \mathrm{E}$.

Cet étiquetage est caractérisé par les trois propriétés suivantes :

- une arête de $\mathcal{T}$ de type $\{0,1\}$, qui dans $D$ est indexée par $a$, est étiquetée $(a, a)$;

- les trois arêtes d'une même face positive sont étiquetées par des éléments de $\mathrm{E} \times \mathrm{E}$ de premières projections égales;

- les trois arêtes d'une même face négative sont étiquetées par des éléments de $\mathrm{E} \times \mathrm{E}$ de deuxièmes projections égales. 
L'allure locale de la triangulation au voisinage d'une arête de type $\{0,1\}$ est représentée par la figure suivante :

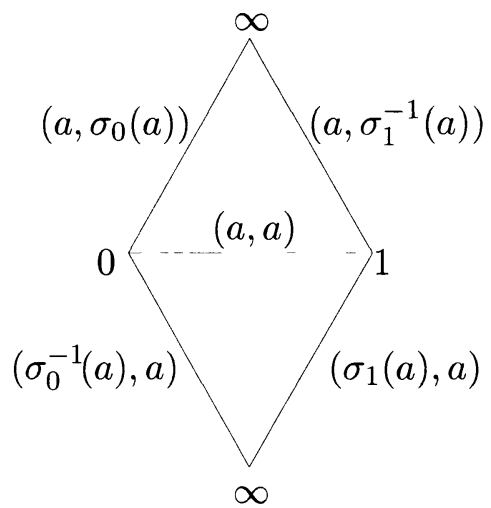

Figure 2

\subsection{Un exemple.}

Considérons le revêtement ramifié représenté par le dessin d'enfant suivant

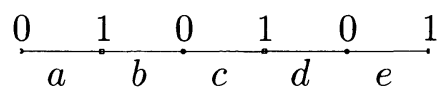

La triangulation correspondante, avec étiquetage des arêtes par des éléments de $\mathrm{E} \times \mathrm{E}$, où $\mathrm{E}=\{a, b, c, d, e\}$, est représentée par la figure ci-dessous (dans laquelle nous avons écrit $x y$ l'élément $(x, y)$ de $\mathrm{E} \times \mathrm{E}$ pour alléger les notations)

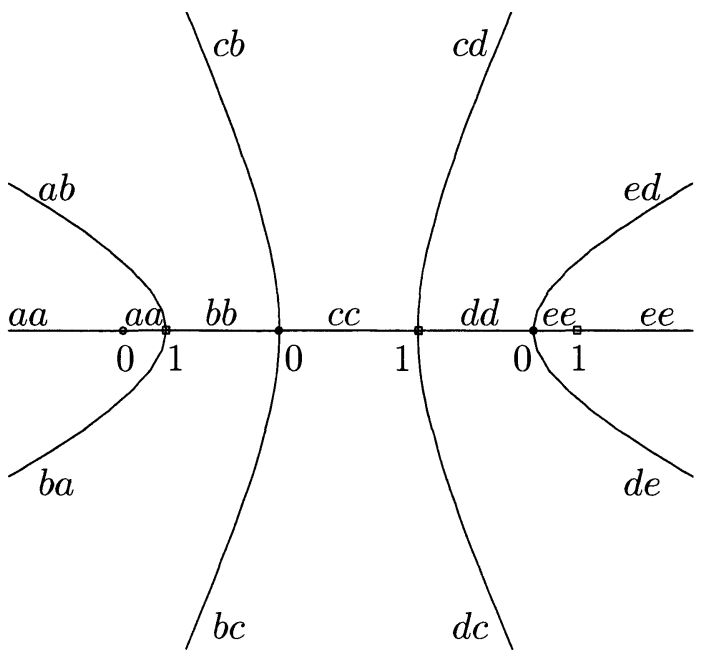

Figure 3 


\section{Composantes connexes de $\widetilde{\Gamma}(\mathbf{R})$}

\subsection{Paramétrisation des composantes connexes de $\widetilde{\Gamma}(\mathbf{R})$.}

La courbe algébrique $\widetilde{\Gamma}$ est projective et lisse. L'ensemble $\widetilde{\Gamma}(\mathbf{R})$ de ses points réels est donc une courbe analytique réelle (lisse) compacte, et ses composantes connexes sont isomorphes (en tant que variétés analytiques réelles) à des cercles. Les points de $\widetilde{\Gamma}(\mathbf{R})$ au-dessus de $0,1, \infty$ et les arêtes de $\widetilde{\Gamma}(\mathbf{R})$ forment une décomposition simpliciale de $\widetilde{\Gamma}(\mathbf{R})$; chaque arête a deux extrémités distinctes et chaque sommet est extrémité d'exactement deux arêtes.

Les arêtes de $\widetilde{\Gamma}(\mathbf{R})$ s'identifient à celles de $\Gamma(\mathbf{R})$, i.e. de la triangulation $\mathrm{T}$ de $\mathrm{X}(\mathbf{C})$. Celles d'un type donné sont paramétrées par $\mathrm{E}$ (via la paramétrisation positive, définie au $\S 2$ ). Appelons demi-arête de $\widetilde{\Gamma}(\mathbf{R})$ tout couple $(\mathrm{A}, \mathrm{Q})$ formé d'une arête $\mathrm{A}$ et de l'une de ses deux extrémités $\mathrm{Q}$ dans $\widetilde{\Gamma}(\mathbf{R})$; on dit que $(\mathrm{A}, \mathrm{Q})$ est la demi-arête d'extrémité $\mathrm{Q}$ de l'arête $\mathrm{A}$.

On paramètre les demi-arêtes de $\widetilde{\Gamma}(\mathbf{R})$ par l'ensemble $\mathrm{F}=\mathrm{E} \times \mathfrak{S}_{\{0,1, \infty\}}$ de la manière suivante : à un élément $(a, s)$ de $\mathrm{F}$ correspond la demiarête $(\mathrm{A}, \mathrm{Q})$, où $\mathrm{A}$ est l'arête de type $\{s(0), s(1)\}$ paramétrée par $a$, et Q l'extrémité de A de type $s(0)$.

On munit l'ensemble des demi-arêtes de $\widetilde{\Gamma}(\mathbf{R})$ de deux involutions : la première associe à une demi-arête l'unique autre demi-arête de même extrémité; la seconde associe à une demi-arête l'autre demi-arête de la même arête. Notons $\tau_{0}$ et $\tau_{1}$ les involutions correspondantes de F. L'ensemble des composantes connexes de $\widetilde{\Gamma}(\mathbf{R})$ est alors paramétré par $\left\langle\tau_{0}, \tau_{1}\right\rangle \backslash \mathrm{F}$. Nous explicitons les involutions $\tau_{0}$ et $\tau_{1}$ de $\mathrm{F}$ au $\S 4.3$.

\subsection{Allure locale des arêtes de $\Gamma(R)$ d'extrémité donnée.}

La définition des demi-arêtes de $\Gamma(\mathbf{R})$ (ou ce qui revient au même de la triangulation $\mathrm{T}$ de $\mathrm{X}(\mathbf{C})$ ) est analogue à celle donnée au $\S 3.1$ pour $\widetilde{\Gamma}(\mathbf{R})$. Les demi-arêtes de $\widetilde{\Gamma}(\mathbf{R})$ et de $\Gamma(\mathbf{R})$ se correspondent bijectivement. Par contre, il se peut que plus de deux demi-arêtes de $\Gamma(\mathbf{R})$ aient une même extrémité dans $\Gamma(\mathbf{R})$.

Soit $\mathrm{P}$ un point de $\mathrm{X}(\mathbf{C})$ au-dessus de 0,1 ou $\infty$, et soit $e$ l'indice de ramification de $f$ en $\mathrm{P}$. Il existe une uniformisante locale $u$ de la surface de Riemann $\mathrm{X}(\mathbf{C})$ au voisinage de $\mathrm{P}$ dans laquelle l'équation locale de $\Gamma(\mathbf{R})$ s'écrit $u^{e}=\bar{u}^{e}([2], \S 2.3 .1$ et 2.3.3). Dans la carte locale correspondante, les germes des demi-arêtes d'extrémité $\mathrm{P}$ s'identifient aux germes des demidroites issues de l'origine engendrées par les racines $2 e$-ièmes de l'unité. L'ensemble des demi-arêtes d'extrémité $\mathrm{P}$ est donc de cardinal $2 e$, et est muni d'un ordre cyclique; deux de ses éléments consécutifs proviennent d'arêtes de type différent (i.e. de type $\{0,1\}$ et $\{0, \infty\}$, si P est de type 0 ). 
Tout antécédent $\mathrm{Q}$ de $\mathrm{P}$ dans $\widetilde{\Gamma}(\mathbf{C})$ est réel ([2], §1.6.7, th. 1), et l'application composée $\widetilde{\Gamma}(\mathbf{R}) \rightarrow \Gamma(\mathbf{R}) \rightarrow \mathrm{X}(\mathbf{C})$ est une immersion (analytique réelle) en $\mathrm{Q}([2], \S 2.3 .3)$. Il en résulte que deux demi-arêtes de $\Gamma(\mathbf{R})$ d'extrémité $\mathrm{P}$ se relèvent en des demi-arêtes d'extrémité commune dans $\widetilde{\Gamma}(\mathbf{R})$ si et seulement si leurs germes dans la carte locale précédente sont ceux de deux demi-droites opposées.

\subsection{Interprétation des involutions $\tau_{0}$ et $\tau_{1}$ en termes de la subdi- vision barycentrique de $\left(\mathrm{E}, \sigma_{0}, \sigma_{1}\right)$.}

Rappelons ([2], B.5.4) que la subdivision barycentrique du dessin combinatoire $\left(\mathrm{E}, \sigma_{0}, \sigma_{1}\right)$ est le dessin combinatoire $\left(\mathrm{F}, \sigma_{0}^{\prime}, \sigma_{1}^{\prime}\right)$, où $\mathrm{F}=\mathrm{E} \times \mathfrak{S}_{\{0,1, \infty\}}$ comme en 4.1 , et où

$$
\begin{aligned}
& \sigma_{0}^{\prime}(x, s)=\left\{\begin{array}{c}
\left(x, s \circ t_{1 \infty}\right) \text { si } s \text { est paire, } \\
\left(\sigma_{s(0)}(x), s \circ t_{1 \infty}\right) \text { si } s \text { est impaire, }
\end{array}\right. \\
& \sigma_{1}^{\prime}(x, s)=\quad\left(x, s \circ t_{01}\right) \text {, }
\end{aligned}
$$

en posant $\sigma_{\infty}=\sigma_{0}^{-1} \sigma_{1}^{-1}$ et en notant $t_{i j}$, pouri, $j \in\{0,1, \infty\}$, la transposition de $\{0,1, \infty\}$ qui échange $i$ et $j$.

Lorsque l'on paramètre l'ensemble des demi-arêtes de $\Gamma(\mathbf{R})$ par $\mathrm{F}, \sigma_{0}^{\prime}$ s'interprète comme la permutation qui à une demi-arête associe la demi-arête de même extrémité qui lui succède immédiatement dans l'ordre cyclique considéré en 4.2. Toute cycle de $\sigma_{0}^{\prime}$ est donc de longueur paire et l'on a, compte tenu de 4.2 ,

$$
\tau_{0}(x, s)=\sigma_{0}^{\prime e}(x, s)
$$

si le cycle de $\sigma_{0}^{\prime}$ contenant $(x, s)$ est de longueur $2 e$ (ce qui équivaut à dire que le cycle de $\sigma_{s(0)}$ contenant $x$ est de longueur $e$ ). Par ailleurs, il est clair que l'on a

$$
\tau_{1}=\sigma_{1}^{\prime} .
$$

Ces formules permettent, compte tenu des numéros 4.1 et 4.2 de déterminer de manière algorithmique à partir du dessin combinatoire $\left(\mathrm{E}, \sigma_{0}, \sigma_{1}\right)$ la liste des composantes connexes de $\widetilde{\Gamma}(\mathbf{R})$, ainsi que la manière dont les demi-arêtes se succèdent sur chacune d'elles.

Remarques. 1) Les arêtes de la triangulation $\mathrm{T}$ (ou de $\Gamma(\mathbf{R})$, ou de $\widetilde{\Gamma}(\mathbf{R})$, cela revient au même) correspondent bijectivement aux éléments de $\left\langle\tau_{1}\right\rangle \backslash \mathrm{F}$ : à la classe $\left\langle\tau_{1}\right\rangle(a, s)$ d'un élément $(a, s)$ de $\mathrm{F}$ correspond l'arête de $\mathrm{T}$ de type $\{s(0), s(1)\}$ paramétrée par $a$. 
2) Les sommets de la triangulation $T$ (ou de $\Gamma(\mathbf{R})$, cela revient au même) correspondent bijectivement aux éléments de $\left\langle\sigma_{0}^{\prime}\right\rangle \backslash \mathrm{F}$ : à la classe $\left\langle\sigma_{0}^{\prime}\right\rangle(a, s)$ d'un élément $(a, s)$ de $\mathrm{F}$ correspond le sommet de $\mathrm{T}$ de type $\{s(0)\}$ paramétré par l'élément $\left\langle\sigma_{s(0)}\right\rangle a$ de $\left\langle\sigma_{s(0)}\right\rangle \backslash \mathrm{E}$.

3) Les sommets de $\widetilde{\Gamma}(\mathbf{R})$ correspondent bijectivement aux éléments de $\left\langle\tau_{0}\right\rangle \backslash \mathrm{F}$ : à la classe $\left\langle\tau_{0}\right\rangle(a, s)$ d'un élément $(a, s)$ de $\mathrm{F}$ correspond le sommet de $\widetilde{\Gamma}(\mathbf{R})$ de type $\{s(0)\}$ paramétré par l'élément $\left\langle\Sigma_{s(0)}\right\rangle(a, a)$ de $\left\langle\Sigma_{s(0)}\right\rangle \backslash(\mathrm{E} \times$ E) si $\{s(0), s(1)\}=\{0,1\}$, par l'élément $\left\langle\Sigma_{s(0)}\right\rangle\left(a, \sigma_{0}(a)\right)$ si $\{s(0), s(1)\}=$ $\{0, \infty\}$ et par l'élément $\left\langle\Sigma_{s(0)}\right\rangle\left(a, \sigma_{1}^{-1}(a)\right)$ si $\{s(0), s(1)\}=\{1, \infty\}$. 4) Soit $(a, s) \in \mathrm{F}$. Soit $e$ la longueur du cycle de $\sigma_{s(0)}$ contenant $a$. La composante connexe de $\widetilde{\Gamma}(\mathbf{R})$ qui contient la demi-arête paramétrée par $(a, s)$ se compose des $2 e$ demi-arêtes paramétrées par les éléments de $\mathrm{F}$ obtenus à partir de $(a, s)$ en appliquant alternativement les involutions $\tau_{0}$ et $\tau_{1}$.

\section{Composantes connexes de l'ensemble des points réels d'une composante irréductible de $\widetilde{\Gamma}$}

Soit C une composante irréductible de $\Gamma$. Si C n'est pas géométriquement irréductible, elle n'a pas de points réels ([2], §2.5.3, prop. 7). Supposons C géométriquement irréductible, et notons $\widetilde{\mathrm{C}}$ sa normalisée. C'est une composante (géométriquement) irréductible de $\widetilde{\mathrm{C}}$ et aussi une composante connexe de $\widetilde{\Gamma}$. Il en résulte que l'ensemble $\widetilde{\mathrm{C}}(\mathbf{R})$ de ses points réels est ouvert et fermé dans $\widetilde{\Gamma}(\mathbf{R})$; il est donc réunion d'un certain nombre de composantes connexes de $\widetilde{\Gamma}(\mathbf{R})$.

L'orbite $\mathcal{O}$ de $\left\langle\Sigma_{0}, \Sigma_{1}\right\rangle$ dans $\mathrm{E} \times \mathrm{E}$ associée à $\mathrm{C}([2], \S 2.1 .1)$ est stable par l'involution $(a, b) \mapsto(b, a)$ de $\mathrm{E} \times \mathrm{E}([2], \S 2.5 .1)$. Notons $\mathrm{F}_{\mathcal{O}}$ l'ensemble des éléments $(a, s)$ de $\mathrm{F}$ possédant l'une des trois propriétés suivantes : soit $\{s(0), s(1)\}=\{0,1\}$ et $(a, a)$ appartient à $\mathcal{O}$; soit $\{s(0), s(1)\}=\{0, \infty\}$ et $\left(a, \sigma_{0}(a)\right)$ appartient à $\mathcal{O}$; soit $\{s(0), s(1)\}=\{1, \infty\}$ et $\left(a, \sigma_{1}^{-1}(a)\right)$ appartient à $\mathcal{O}$.

Il résulte de $\S 3.4$ et de $\S 3.1$ que $\mathrm{F}_{\mathcal{O}}$ paramètre les demi-arêtes de $\Gamma(\mathbf{R})$ contenues dans $\mathrm{C}(\mathbf{R})$ (ou ce qui revient au même les demi-arêtes de $\widetilde{\Gamma}(\mathbf{R})$ contenues dans $\widetilde{\mathrm{C}}(\mathbf{R})$ ). L'ensemble des composantes connexes de $\widetilde{\mathrm{C}}(\mathbf{R})$ est donc paramétré par $\left\langle\tau_{0}, \tau_{1}\right\rangle \backslash \mathrm{F}_{\mathcal{O}}$. Nous savons déjà que cet ensemble peut être vide (cf. [2], §2.5.3). Nous montrerons au numéro suivant par un exemple qu'il peut être de cardinal $\geq 2$.

\section{Un exemple où l'ensemble des points réels d'une composante irréductible de $\widetilde{\Gamma}$ n'est pas connexe}

\subsection{Description de l'exemple, par un dessin combinatoire et le dessin d'enfant associé.}

Considérons le dessin combinatoire $\left(\mathrm{E}, \sigma_{0}, \sigma_{1}\right)$, où 


$$
\begin{aligned}
\mathrm{E} & =\{a, b, c, d, e, f, g, h, j\}, \\
\sigma_{0} & =(a b c d)(e)(f g)(h j) \\
\sigma_{1} & =(a)(b e d f)(c)(g h)(j) .
\end{aligned}
$$

On a $\sigma_{\infty}=\sigma_{0}^{-1} \sigma_{1}^{-1}=(a d e)(b g j h f c)$.

Le dessin combinatoire (E, $\left.\sigma_{0}, \sigma_{1}\right)$ est donc connexe, de genre 0 , et peut être représenté par le dessin d'enfant suivant :

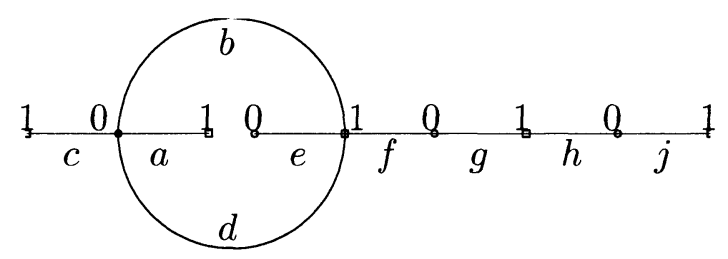

Figure 4

Pour l'action du groupe $\left\langle\Sigma_{0}, \Sigma_{1}\right\rangle, \mathrm{E} \times \mathrm{E}$ possède trois orbites $\mathcal{O}_{1}, \mathcal{O}_{2}, \mathcal{O}_{3}$, sur lesquelles $\Sigma_{0}, \Sigma_{1}$ et $\Sigma_{\infty}=\Sigma_{0}^{-1} \Sigma_{1}^{-1}$ opèrent comme suit (en écrivant $x y$ l'élément $(x, y)$ de $\mathrm{E} \times \mathrm{E}$ pour alléger les notations)

$$
\begin{aligned}
& \Sigma_{0} \mid \mathcal{O}_{1}=\quad(a a, b d, c c, d b)(e e)(f f, g g)(h h, j j), \\
& \Sigma_{1} \mid \mathcal{O}_{1}=\quad(a a)(b d, e e, d b, f f)(c c)(g g, h h)(j j), \\
& \Sigma_{\infty} \mid \mathcal{O}_{1}=\quad(a a, d b, e e)(b d, g g, j j, h h, f f, c c), \\
& \Sigma_{0} \mid \mathcal{O}_{2}=(a b, b a, c d, d c)(a d, b c, c b, d a)(a f, b g, c f, d g)(a e, b e, c e, d e) \\
& (g h, f j)(j f, h g)(e a, e d, e c, e b)(f c, g b, f a, g d)(f h, g j) \\
& (h j, j h)(j g, h f)(h e, j e)(e h, e j)(d f, a g, b f, c g) \\
& (f d, g c, f b, g a)(c h, d j, a h, b j)(j b, h a, j d, h c) \text {, } \\
& \Sigma_{1} \mid \mathcal{O}_{2}=(a b, a f, a d, a e)(b a, e a, d a, f a)(d c, f c, b c, e c)(c d, c e, c b, c f) \\
& (d g, f h, b g, e h)(h g, g h)(b e, e b, d f, f d)(e d, d e, f b, b f)(g j, h j)) \\
& (j h, j g)(h f, g d, h e, g b)(j e, j b, j f, j d)(a g, a h)(e j, d j, f j, b j) \\
& (c g, c h)(g c, h c)(g a, h a) \text {, } \\
& \Sigma_{\infty} \mid \mathcal{O}_{2}=\quad(a b, d e, e a)(a d, d g, e j, a h, d f, e c)(a e, d a, e b) \\
& (a f, d c, e d, a g, d j, e h)(b a, g b, j e, h a, f b, c e) \\
& (b g, g j, j h, h f, f c, c d)(b c, g d, j g, h j, f h, c f) \\
& (b e, g a, j b, h e, f a, c b)(b f, g c, j d, h g, f j, c h) \\
& (b j, g h, j f, h c, f d, c g) \text {, } \\
& \Sigma_{0} \mid \mathcal{O}_{3}=(a c, b b, c a, d d)(e f, e g)(f e, g e)(d h, a j, b h, c j)(f g, g f) \\
& (h b, j a, h d, j c)
\end{aligned}
$$




$$
\begin{array}{cc}
\Sigma_{1} \mid \mathcal{O}_{3}= & (a c)(b b, e f, d d, f e)(c a)(e g, d h, f g, b h)(g e, h b, g f, h d) \\
(a j)(c j)(j a)(j c), & \\
\Sigma_{\infty} \mid \mathcal{O}_{3}= & (a c, d d, e g, a j, d h, e f)(b b, g e, j a, h b, f e, c a) \\
(b h, g f, j c, h d, f g, c j) .
\end{array}
$$

Ces orbites sont stables par l'involution $x y \mapsto y x$. Elles correspondent donc à trois composantes irréductibles de $\Gamma_{(\mathbf{C})}$, toutes réelles, i.e. à trois composantes géométriquement irréductibles $\mathrm{C}_{1}, \mathrm{C}_{2}, \mathrm{C}_{3}$ de $\Gamma$. Celles-ci sont de genres 0,6 et 1 respectivement.

Une triangulation adaptée du dessin d'enfant de la figure 4 est représentée sur la figure 5 (qu'il convient de considérer comme complétée par un point à l'infini en lequel se rejoignent les branches infinies ; deux demi-arêtes aboutissant en ce point sont opposées si les branches infinies correspondantes ont des directions asymptotiques opposées). L'examen de cette figure permet, compte tenu de 4.2 , de conclure que $\widetilde{\Gamma}(\mathbf{R})$ possède quatre composantes connexes, dont les images dans $\Gamma(\mathbf{R})$ sont numérotées (1), (2), (3), (4) sur la figure ci-dessous.

(2)

(2)

(1)

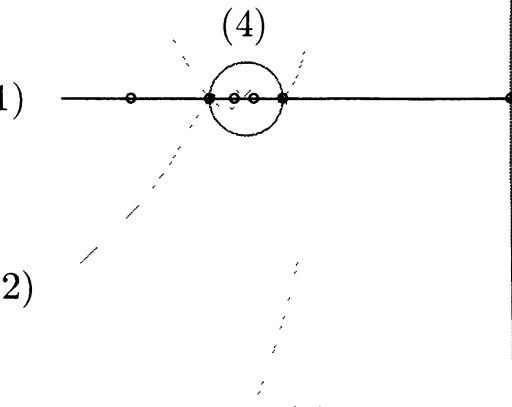

(2)
(3)

(2)

(2)

(3)

\section{(2)}

Figure 5

L'étiquetage des arêtes de cette triangulation par des éléments de $\mathrm{E} \times \mathrm{E}$, suivant la méthode décrite au $\S 3.7$, est la suivante : 


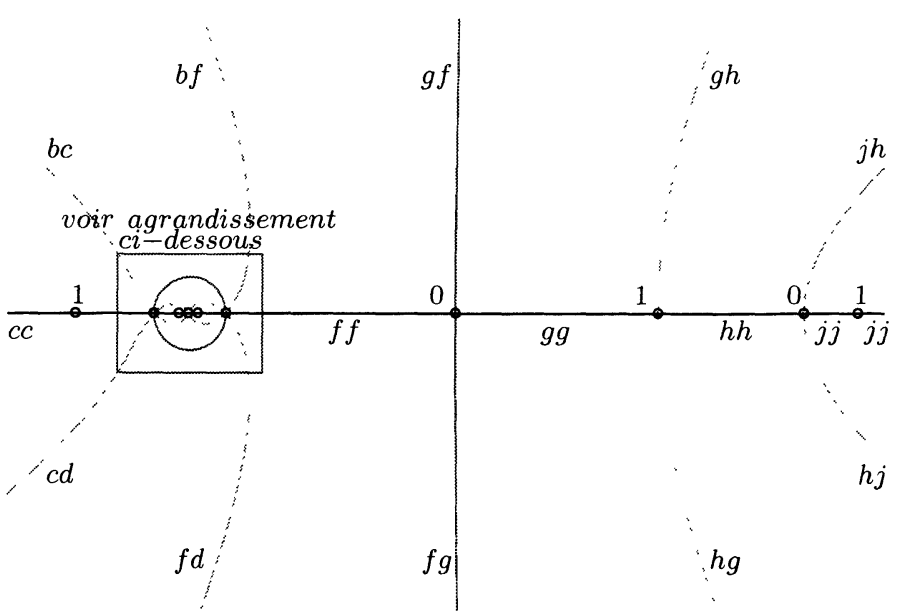

Figure 6

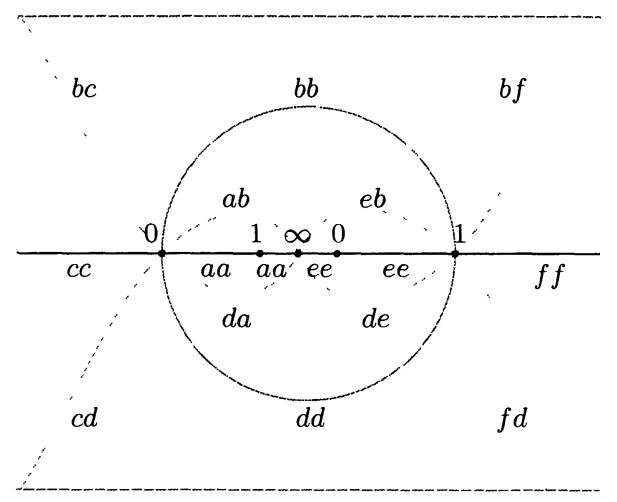

Figure 7

Il résulte du $\S 5$ que $\widetilde{\mathrm{C}}_{1}(\mathbf{R})$ est la composante connexe de $\widetilde{\Gamma}(\mathbf{R})$ numérotée (1) sur la figure 1 , que $\widetilde{\mathrm{C}}_{2}(\mathbf{R})$ est la composante connexe de $\widetilde{\Gamma}(\mathbf{R})$ numérotée (2), et que $\widetilde{\mathrm{C}}_{3}(\mathbf{R})$ n'est pas connexe, mais est la réunion des deux composantes connexes de $\widetilde{\Gamma}(\mathbf{R})$ numérotées (3) et (4).

\subsection{Description de l'exemple par une fonction de Belyi.}

Considérons la fonction rationnelle $f$ définie par

(5) $f(z)=\frac{2 z^{4}(z+\alpha-1)\left((1-2 \alpha) z+\alpha^{2}-1\right)^{2}((1-2 \alpha) z+3 \alpha-3)^{2}}{(1-2 \alpha)(3 z-\alpha-1)^{3}}$,

où $\alpha$ est la plus petite des deux racines réelles de l'équation $2 \alpha^{4}-4 \alpha^{3}-$ $2 \alpha+1=0$, c'est-à-dire 
(6)

$$
\alpha=\frac{1}{2}\left(1+\sqrt{1+3^{1 / 3}}-\sqrt{2-3^{1 / 3}+\frac{4}{\sqrt{1+3^{1 / 3}}}}\right) \approx 0,3985898695 \ldots
$$

On a

(7) $f(z)-1=\frac{(z-1)^{4}((1-2 \alpha) z-\alpha-1)^{2}\left(2(1-2 \alpha) z^{3}+6(\alpha-1) z^{2}-3 z+\alpha+1\right)}{(3 z-\alpha-1)^{3}}$.

Le degré de $f$ est 9 et 0,1 et $\infty$ ont respectivement 4,5 et 2 antécédents par $f$ dans $\mathbf{P}^{1}$. Comme $(4+5+2)-9=2, f$ est une fonction de Belyi, i.e. définit un revêtement ramifié $\mathbf{P}^{1} \rightarrow \mathbf{P}^{1}$, non ramifié au-dessus de $\mathbf{P}^{1}-$ $\{0,1, \infty\}$. Le dessin combinatoire qui lui est associé est isomorphe au dessin combinatoire $\left(\mathrm{E}, \sigma_{0}, \sigma_{1}\right)$ considéré au $\S 6.1,(4)$ : cela se déduit du fait qu'il est défini sur $\mathbf{R}$, que les points au-dessus de 0,1 et $\infty$ sont réels, qu'ils ont mêmes valences et se succèdent sur la droite réelle dans le même ordre que ceux de la figure 6 .

Les orbites de $\sigma_{0}$ dans E paramètrent les points de $f^{-1}(0)$ : aux orbites $\{a, b, c, d\},\{e\},\{f, g\},\{h, j\}$ correspondent respectivement les points 0 , $1-\alpha, \frac{1-\alpha^{2}}{1-2 \alpha}, \frac{3(1-\alpha)}{1-2 \alpha}$. Les orbites de $\sigma_{1}$ dans E paramètrent les points de $f^{-1}(1)$ : aux orbites $\{b, e, d, f\}$ et $\{g, h\}$ correspondent respectivement les points 1 et $\frac{1+\alpha}{1-2 \alpha}$, et aux orbites $\{c\}\{a\},\{j\}$ les trois racines (toutes réelles) du polynôme $2(1-2 \alpha) z^{3}+6(\alpha-1) z^{2}-3 z+\alpha+1$, rangées par ordre croissant. Les orbites de $\sigma_{\infty}$ dans E paramètrent les points de $f^{-1}(\infty)$ : aux orbites $\{a, d, e\}$ et $\{b, g, j, h, f, c\}$ correspondent respectivement les points $\frac{1+\alpha}{3}$ et $\infty$.

Proposition 6.1. L'ouvert affine $\mathrm{U}=\prod_{\mathbf{C} / \mathbf{R}} \mathbf{A}^{1}$ de $\prod_{\mathbf{C} / \mathbf{R}} \mathbf{P}^{1}$ s'identifie canoniquement à un plan muni de coordonnées $x, y$. La trace de $\mathrm{C}_{3}$ sur $\mathrm{U}$ est la courbe affine d'équation

$$
x(x-1)\left(x-\frac{1-\alpha^{2}}{1-2 \alpha}\right)+y^{2}\left(x-\frac{(2-\alpha)^{2}}{3(1-2 \alpha)}\right)=0 .
$$

On obtient une équation de la trace de $\Gamma$ sur $U$ en égalisant le numérateur de $\operatorname{Im}(f(x+i y))$ avec 0 . Cette équation est polynomiale de degré 12 . Son premier membre est produit de 3 facteurs irréductibles, de degrés 1,8 et 3 , qui sont les équations des traces sur $\mathrm{U}$ de $\mathrm{C}_{1}, \mathrm{C}_{2}$ et $\mathrm{C}_{3}$ respectivement. La proposition peut alors se démontrer en effectuant cette factorisation. C'est a priori difficile sans ordinateur; c'est pourquoi nous allons présenter une autre démonstration de la prop. 6.1 nécessitant moins de calculs. 
On sait a priori que la courbe $\mathrm{C}_{3}$ est lisse $([2], \S .2 .4 .4$, th. 2$)$ et que sa trace sur $\mathrm{U}$ est une cubique ( $\$ 7.2 .4$, exemple 2 ) symétrique par rapport à l'axe $y=0$. Son équation est donc de la forme $\mathrm{P}(x)+y^{2} \mathrm{Q}(x)$, avec $\operatorname{deg}(\mathrm{P}) \leq 3$ et $\operatorname{deg}(\mathrm{Q}) \leq 1$; en la multipliant par une constante, on se ramène au cas où le polynôme $\mathrm{P}$ est unitaire. Les racines de $\mathrm{P}$ sont les abscisses des points d'intersection de $\mathrm{C}_{3} \cap \mathrm{U}$ avec l'axe $y=0$; on a donc $\mathrm{P}(x)=x(x-1)\left(x-\frac{1-\alpha^{2}}{1-2 \alpha}\right)$.

Les orbites de $\Sigma_{0}$ dans $\mathcal{O}_{3}$ paramètrent les points complexes de $\widetilde{\mathrm{C}}_{3}=\mathrm{C}_{3}$ au-dessus de 0 . Considérons en particulier les deux points correspondant aux orbites $\{e f, e g\}$ et $\{d h, a j, b h, c j\}$. Ce sont les points $\left(1-\alpha, \frac{1-\alpha^{2}}{1-2 \alpha}\right)$ et $\left(0, \frac{3(1-\alpha)}{1-2 \alpha}\right)$ de $\mathbf{P}^{1} \times \mathbf{P}^{1}$ lorsque l'on plonge la complexifiée de $\mathrm{C}_{3}$ dans $\mathrm{X} \times \mathrm{X}^{*} \approx \mathbf{P}^{1} \times \mathbf{P}^{1}$. Ce plongement (ou plus exactement la restriction de ce plongement à $\left.\mathrm{C}_{3} \cap \mathrm{U}\right)$ s'écrit $(x, y) \mapsto(x+i y, x-i y)$. Les coordonnées $(x, y)$ des deux points considérés sont donc $\left(\frac{(1-\alpha)(2-\alpha)}{2(1-2 \alpha)}, \frac{3 \alpha(1-\alpha) i}{2(1-2 \alpha)}\right)$ et $\left(\frac{3(1-\alpha)}{2(1-2 \alpha)}, \frac{3(1-\alpha) i}{2(1-2 \alpha)}\right)$. En écrivant que ces points satisfont l'équation de $\mathrm{C}_{3}$, on obtient les relations

$$
\begin{aligned}
\mathrm{Q}\left(\frac{(1-\alpha)(2-\alpha)}{2(1-2 \alpha)}\right) & =\frac{(\alpha+1)(\alpha-2)}{6(1-2 \alpha)}, \\
\mathrm{Q}\left(\frac{3(1-\alpha)}{2(1-2 \alpha)}\right) & =\frac{(\alpha+1)(1-2 \alpha)}{6(1-2 \alpha)},
\end{aligned}
$$

d'où il résulte que $\mathrm{Q}(x)=x-\frac{(\alpha-2)^{2}}{3(1-2 \alpha)}$.

Remarque. Pour déterminer Q, on peut aussi utiliser les arguments suivants. Soit C l'adhérence de $\mathrm{C}_{3} \cap \mathrm{U}$ dans $\mathbf{P}^{2}$. C'est une cubique projective dont la normalisée est de genre 1 ; elle est donc lisse et isomorphe à $\widetilde{\mathrm{C}}_{3}=\mathrm{C}_{3}$. On peut démontrer directement qu'elle possède trois points à l'infini, qui sont $(0,1,0),(1, i, 0)$ et $(1,-i, 0)$; cela implique que la partie homogène de degré 3 de l'équation de $\mathrm{C}_{3} \cap \mathrm{U}$ est $x\left(x^{2}+y^{2}\right)$, donc que le polynôme $\mathrm{Q}$ est unitaire. La racine $\beta$ de $\mathrm{Q}$ est l'abscisse de l'asymptote verticale de $\mathrm{C}_{3} \cap \mathrm{U}$. Lorsqu'un point $(x, y)$ de $\mathrm{C}_{3} \cap \mathrm{U}$, avec $x, y$ réels, se déplace sur la branche asymptotique en tendant vers l'infini, $x$ tend vers $\beta$ et $|y|$ vers $+\infty$; on a donc

$$
\begin{aligned}
\frac{27 f(x+i y)}{2(1-2 \alpha)^{3}} & =(i y)^{6}+(i y)^{5}\left(6 x-\left(1-\alpha+2 \frac{1-\alpha^{2}}{1-2 \alpha}+2 \frac{3(1-\alpha)}{1-2 \alpha}-3 \frac{1+\alpha}{3}\right)\right)+o\left(y^{5}\right) \\
& =-y^{6}+6 i y^{5}\left(\beta-\frac{(\alpha-2)^{2}}{3(1-2 \alpha)}\right)+o\left(y^{5}\right),
\end{aligned}
$$

d'où $\beta=\frac{(\alpha-2)^{2}}{3(1-2 \alpha)}$ puisque $f(x+i y)$ est réel. 


\subsection{Détermination de la fonction de Belyi $f$ via l'étude de $\mathrm{C}_{3}$.}

$\mathrm{Au}$ numéro précédent, nous avions introduit ex abrupto une fonction rationnelle $f$ et vérifié qu'elle était la fonction de Belyi de l'exemple considéré (normalisée de sorte que 0 soit le zéro d'ordre 4 de $f, 1$ le zéro d'ordre 4 de $f-1$ et $\infty$ le pôle d'ordre 6 de $f$ ), mais nous avions pas indiqué comment nous l'avions trouvée. Il existe des algorithmes pour ce faire, mais ils conduisent à des calculs assez fastidieux, difficiles à mener sans l'aide d'un ordinateur.

Nous allons dans ce numéro montrer comment l'étude géométrique de la seule composante irréductible $\mathrm{C}_{3}$ de $\Gamma$ permet de déterminer simplement $f$. La fonction de Belyi recherchée est de la forme

$$
f(z)=\frac{\lambda z^{4}\left(z-\alpha_{0}\right)\left(z-\alpha_{1}\right)^{2}\left(z-\alpha_{2}\right)^{2}}{(z-\gamma)^{3}},
$$

avec

$$
f(z)-1=\frac{\lambda(z-1)^{4}\left(z-\beta_{0}\right)^{2}\left(z-\beta_{1}\right)\left(z-\beta_{2}\right)\left(z-\beta_{3}\right)}{(z-\gamma)^{3}},
$$

les nombres $\alpha_{i}, \beta_{j}, \gamma$ et $\lambda$ étant réels et tels que

$$
\beta_{1}<0<\beta_{2}<\gamma<\alpha_{0}<1<\alpha_{1}<\beta_{0}<\alpha_{2}<\beta_{3} .
$$

D'après la démonstration de la prop. 2 et la remarque qui la suit, la trace de $\mathrm{C}_{3}$ sur le plan affine $\mathrm{U}$ a pour équation

$$
x(x-1)\left(x-\alpha_{1}\right)+y^{2}(x-\delta)=0
$$

où $\delta=\left(\alpha_{0}+2 \alpha_{1}+2 \alpha_{2}-3 \gamma\right) / 6$. L'adhérence de $\mathrm{C}_{3} \cap \mathrm{U}$ dans le plan projectif est un modèle projectif plan de $\mathrm{C}_{3}$; son équation s'écrit, en notant $\underline{x}, \underline{y}, \underline{t}$ les coordonnées homogènes,

$$
\underline{x}(\underline{x}-\underline{t})\left(\underline{x}-\alpha_{1} \underline{t}\right)+\underline{y}^{2}(\underline{x}-\delta \underline{t})=0 .
$$

La restriction de $g_{(\mathbf{C})}$ à la complexifiée de $\mathrm{C}_{3}$ est un revêtement ramifié de $\mathbf{P}^{1}$, non ramifié au-dessus de $\mathbf{P}^{1}-\{0,1, \infty\}$. Le dessin combinatoire associé s'identifie à $\left(\mathcal{O}_{3}, \Sigma_{0}\left|\mathcal{O}_{3}, \Sigma_{1}\right| \mathcal{O}_{3}\right)$. Vu les formules donnant $\Sigma_{0}\left|\mathcal{O}_{3}, \Sigma_{1}\right| \mathcal{O}_{3}$ et $\Sigma_{\infty} \mid \mathcal{O}_{3}$ (cf. $\S 6.1$ ), le dessin d'enfant associé peut se représenter comme suit (la surface de genre 1 sur laquelle il est tracé étant obtenue en identifiant les côtés opposés du carré). 


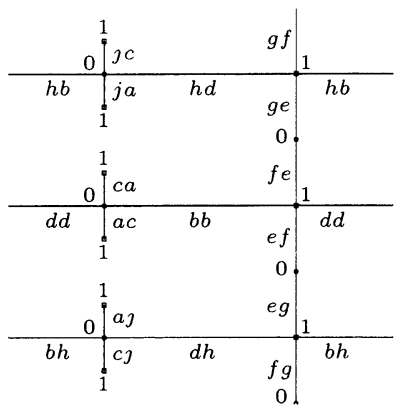

Figure 8

On constate qu'un groupe cyclique d'ordre 3 de translations verticales opère sans points fixes sur ce dessin. Le groupe correspondant d'automorphismes du dessin combinatoire est engendré par la permutation

$$
\tau=(a c, j a, c j)(b b, h d, d h)(c a, j c, a j)(d d, h b, b h)(e f, g e, f g)(f e, g f, e g)
$$

de $\mathcal{O}_{3}$ (qui commute à $\Sigma_{0} \mid \mathcal{O}_{3}$ et à $\Sigma_{1} \mid \mathcal{O}_{3}$ et opère sans points fixes dans l'ensemble des orbites de $\Sigma_{0} \mid \mathcal{O}_{3}$, de $\Sigma_{1} \mid \mathcal{O}_{3}$ et de $\left.\Sigma_{\infty} \mid \mathcal{O}_{3}\right)$. Notons $\mathrm{G}$ le groupe correspondant d'automorphismes du revêtement $g_{(\mathbf{C})}$; il est stable par la conjugaison complexe : le conjugué d'un élément de $\mathrm{G}$ est égal à son inverse. On peut donc définir la courbe algébrique réelle quotient $\mathrm{C}=\mathrm{G} \backslash \mathrm{C}_{3}$; elle est de genre 1 . Le morphisme canonique $\pi: \mathrm{C}_{3} \rightarrow \mathrm{C}$ est non ramifié, et le morphisme $g: \mathrm{C}_{3} \rightarrow \mathbf{P}_{\mathbf{R}}^{1}$, de degré 18 , est le composé de $\pi$ et d'un revêtement ramifié $h: \mathrm{C} \rightarrow \mathbf{P}_{\mathbf{R}}^{1}$, de degré 6 , non ramifié au-dessus de $\mathbf{P}_{\mathbf{R}}^{1}-\{0,1, \infty\}$.

Le dessin combinatoire associé au revêtement ramifié $(\mathrm{C}, h)$ est canoniquement isomorphe à $\left(\mathrm{E}^{\prime}, \sigma_{0}^{\prime}, \sigma_{1}^{\prime}\right)$, où $\mathrm{E}^{\prime}$ est l'ensemble $\langle\tau\rangle \backslash \mathrm{E}$ des orbites de $\tau$ dans $\mathrm{E}$, et où les permutations $\sigma_{0}^{\prime}, \sigma_{1}^{\prime}$ de $\mathrm{E}^{\prime}$ sont déduites de $\sigma_{0}, \sigma_{1}$ par passage au quotient. Posons

$$
\begin{array}{ll}
a^{\prime}=\{a c, j a, c j\}, & b^{\prime}=\{b b, h d, d h\}, \quad c^{\prime}=\{c a, j c, a j\}, \\
d^{\prime}=\{d d, h b, b h\}, & e^{\prime}=\{e f, g e, f g\}, \quad f^{\prime}=\{f e, g f, e g\} .
\end{array}
$$

On a alors

$$
\begin{aligned}
\mathrm{E}^{\prime} & =\left\{a^{\prime}, b^{\prime}, c^{\prime}, d^{\prime}, e^{\prime}, f^{\prime}\right\} \\
\sigma_{0}^{\prime} & =\left(a^{\prime} b^{\prime} c^{\prime} d^{\prime}\right)\left(e^{\prime} f^{\prime}\right), \\
\sigma_{1}^{\prime} & =\left(a^{\prime}\right)\left(b^{\prime} e^{\prime} d^{\prime} f^{\prime}\right)\left(c^{\prime}\right), \\
\sigma_{\infty}^{\prime} & =\sigma_{0}^{\prime-1} \sigma_{1}^{\prime-1}=\left(a^{\prime} d^{\prime} f^{\prime} c^{\prime} b^{\prime} e^{\prime}\right) .
\end{aligned}
$$

Puisque le revêtement $(\mathrm{C}, h)$ est défini sur $\mathbf{R}$, la conjugaison complexe définit une involution de $\mathrm{E}^{\prime}$ : celle-ci est $\left(a^{\prime} c^{\prime}\right)\left(b^{\prime}\right)\left(d^{\prime}\right)\left(e^{\prime} f^{\prime}\right)$.

Le dessin d'enfant associé peut se représenter comme suit (la surface de genre 1 sur laquelle il est tracé étant là encore obtenue en identifiant les côtés opposés du carré). 


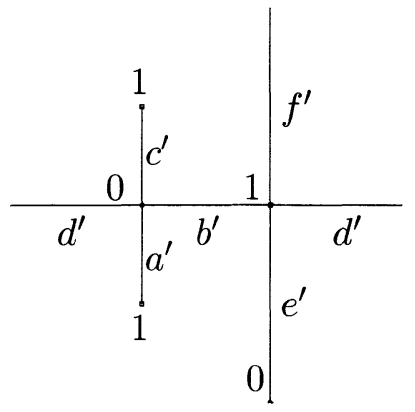

Figure 9

On constate qu'il possède un automorphisme d'ordre 2, déduit de l'involution

$$
\tau^{\prime}=\left(a^{\prime} c^{\prime}\right)\left(b^{\prime} d^{\prime}\right)\left(e^{\prime} f^{\prime}\right)
$$

de $\mathrm{E}^{\prime}$ (qui commute à $\sigma_{0}^{\prime}$ et à $\sigma_{1}^{\prime}$ ). Cette involution stabilise chacune des deux orbites de $\sigma_{0}^{\prime}$, l'orbite à quatre éléments de $\sigma_{1}^{\prime}$ et l'unique orbite de $\sigma_{\infty}^{\prime}$; elle échange les deux orbites de cardinal 1 de $\sigma_{1}^{\prime}$. L'automorphisme d'ordre 2 correspondant de $\mathrm{C}$ est défini sur $\mathbf{R}$. Soit $\mathrm{C}^{\prime}$ la courbe quotient de $\mathrm{C}$ par cet automorphisme. Elle est de genre 0 . La surjection canonique $\pi^{\prime}: \mathrm{C} \rightarrow \mathrm{C}^{\prime}$ est un revêtement de degré 2 , ramifié en quatre points, et $h$ est le composé de $\pi^{\prime}$ et d'un revêtement ramifié $k: \mathrm{C}^{\prime} \rightarrow \mathbf{P}_{\mathbf{R}}^{1}$, de degré 3 , non ramifié au-dessus de $\mathbf{P}_{\mathbf{R}}^{1}-\{0,1, \infty\}$.

Le dessin combinatoire associé au revêtement ramifié $\left(\mathrm{C}^{\prime}, k\right)$ est canoniquement isomorphe à $\left(\mathrm{E}^{\prime \prime}, \sigma_{0}^{\prime \prime}, \sigma_{1}^{\prime \prime}\right)$, où $\mathrm{E}^{\prime \prime}$ est l'ensemble $\left\langle\tau^{\prime}\right\rangle \backslash \mathrm{E}^{\prime}$ des orbites de $\tau^{\prime}$ dans $\mathrm{E}^{\prime}$, et où les permutations $\sigma_{0}^{\prime \prime}, \sigma_{1}^{\prime \prime}$ de $\mathrm{E}^{\prime \prime}$ sont déduites de $\sigma_{0}^{\prime}, \sigma_{1}^{\prime}$ par passage au quotient. Posons

$$
a^{\prime \prime}=\left\{a^{\prime}, c^{\prime}\right\}, \quad b^{\prime \prime}=\left\{b^{\prime}, d^{\prime}\right\}, \quad c^{\prime \prime}=\left\{e^{\prime}, f^{\prime}\right\} .
$$

On a alors

$$
\begin{gathered}
\mathrm{E}^{\prime \prime}=\left\{a^{\prime \prime}, b^{\prime \prime}, c^{\prime \prime}\right\}, \\
\sigma_{0}^{\prime \prime}=\left(a^{\prime \prime} b^{\prime \prime}\right)\left(c^{\prime \prime}\right), \quad \sigma_{1}^{\prime \prime}=\left(a^{\prime \prime}\right)\left(b^{\prime \prime} c^{\prime \prime}\right), \quad \sigma_{\infty}^{\prime \prime}=\sigma_{0}^{\prime \prime-1} \sigma_{1}^{\prime \prime-1}=\left(a^{\prime \prime} b^{\prime \prime} c^{\prime \prime}\right),
\end{gathered}
$$

et la conjugaison complexe opère sur $E^{\prime \prime}$ suivant l'identité.

\section{Remarque 1. L'involution}

$$
(a c, c a)(a j, j a)(b b, d d)(e f, f e),(e g, g e)(f g, g f)(j c, c j)(d h, h b)(h d, b h)
$$

de $\mathrm{E}$ commute à $\sigma_{0}, \sigma_{1}$ et à la conjugaison complexe, et est un relèvement de l'involution $\tau^{\prime}$ de $\left(\mathrm{E}^{\prime}, \sigma_{0}^{\prime}, \sigma_{1}^{\prime}\right)$. Il lui correspond donc un automorphisme d'ordre 2 de $\left(\mathrm{C}_{3}, g\right)$. Celui-ci engendre avec $\mathrm{G}$ un groupe $\mathrm{H}$ d'automorphismes de la complexifiée de $\mathrm{C}_{3}$, stable par conjugaison complexe et isomorphe à $\mathfrak{S}_{3}$. Le morphisme $\pi^{\prime} \circ \pi: \mathrm{C}_{3} \rightarrow \mathrm{C}^{\prime}$ définit par passage au quotient un isomorphisme de $\mathrm{H} \backslash \mathrm{C}_{3}$ sur $\mathrm{C}^{\prime}$. 
Le dessin d'enfant associé au revêtement ramifié $\left(\mathrm{C}^{\prime}, k\right)$ peut se représenter comme suit

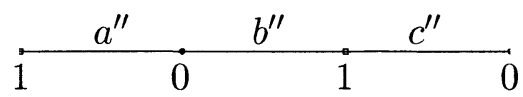

Figure 10

La courbe $\mathrm{C}^{\prime}$ possède au-dessus de chacun des points 0,1 et $\infty$ un unique point en lequel $k$ est ramifié. On identifiera désormais $\mathrm{C}^{\prime}$ à $\mathbf{P}_{\mathbf{R}}^{1}$ par l'isomorphisme qui applique les trois points précédents de $\mathrm{C}^{\prime}$ sur 0,1 et $\infty$ respectivement. Le morphisme $k$ n'est alors autre que la fonction de Belyi

$$
k(z)=-z^{2}(2 z-3),
$$

on a

$$
k(z)-1=-(z-1)^{2}(2 z+1),
$$

et les quatre points de $\mathrm{C}^{\prime}$ représentés sur la figure 10 sont respectivement $-\frac{1}{2}, 0,1, \frac{3}{2}$. Le morphisme $\pi^{\prime}: \mathrm{C} \rightarrow \mathrm{C}^{\prime}$ est ramifié au-dessus des points 0 , $1, \frac{3}{2}, \infty$ de $\mathrm{C}^{\prime}$, et non ramifié ailleurs.

Le morphisme composé $\pi^{\prime} \circ \pi: \mathrm{C}_{3} \rightarrow \mathrm{C}^{\prime}$ s'interprète, lorsque $\mathrm{C}^{\prime}$ est identifiée à $\mathbf{P}_{\mathbf{R}}^{1}$, comme une fonction rationnelle sur $\mathrm{C}_{3}$, que nous noterons $\mathrm{X}$. Son diviseur est

$$
\operatorname{div}(\mathrm{X})=2 \mathrm{~A}_{0}+2 \mathrm{~A}_{1}+2 \mathrm{~A}_{2}-2 \mathrm{D}_{0}-2 \mathrm{D}_{1}-2 \mathrm{D}_{2},
$$

où $\mathrm{A}_{0}, \mathrm{~A}_{1}, \mathrm{~A}_{2}$ sont les sommets de type 0 de $\mathrm{C}_{3}(\mathbf{C})$ paramétrés par les orbites $\{a c, b b, c a, d d\},\{h b, j a, h d, j c\},\{d h, a j, b h, c j\}$ de $\Sigma_{0}$ dans $\mathcal{O}_{3}$ respectivement et $\mathrm{D}_{0}, \mathrm{D}_{1}, \mathrm{D}_{2}$ les sommets de type $\infty$ paramétrés par les orbites $\{b h, g f, j c, h d, f g, c j\},\{a c, d d, e g, a j, d h, e f\},\{b b, g e, j a, h b, f e, c a\}$ de $\Sigma_{\infty}$ (cf. figure 11 ci-dessous). Les coordonnées homogènes de ces points dans le modèle projectif plan de $\mathrm{C}_{3}$ introduit au début de ce numéro sont

$$
\begin{gathered}
\mathrm{A}_{0}=(0,0,1), \quad \mathrm{A}_{1}=\left(\frac{\alpha_{2}}{2},-\frac{i \alpha_{2}}{2}, 1\right), \quad \mathrm{A}_{2}=\left(\frac{\alpha_{2}}{2}, \frac{i \alpha_{2}}{2}, 1\right) \\
\mathrm{D}_{0}=(0,1,0), \quad \mathrm{D}_{1}=(1, i, 0), \quad \mathrm{D}_{2}=(1,-i, 0) .
\end{gathered}
$$




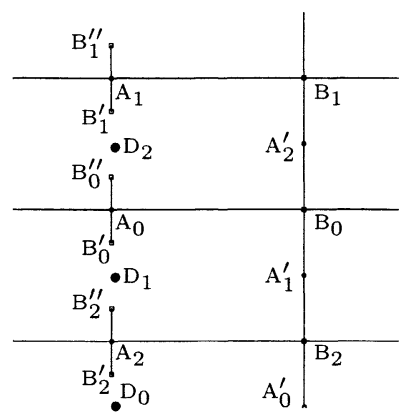

Figure 11

On a de même

$$
\begin{aligned}
& \operatorname{div}(\mathrm{X}-1)=2 \mathrm{~B}_{0}+2 \mathrm{~B}_{1}+2 \mathrm{~B}_{2}-2 \mathrm{D}_{0}-2 \mathrm{D}_{1}-2 \mathrm{D}_{2}, \\
& \operatorname{div}\left(\mathrm{X}-\frac{3}{2}\right)=2 \mathrm{~A}_{0}^{\prime}+2 \mathrm{~A}_{1}^{\prime}+2 \mathrm{~A}_{2}^{\prime}-2 \mathrm{D}_{0}-2 \mathrm{D}_{1}-2 \mathrm{D}_{2},
\end{aligned}
$$

où $\mathrm{A}_{0}^{\prime}, \mathrm{A}_{1}^{\prime}, \mathrm{A}_{2}^{\prime}$ sont les sommets de type 0 de $\mathrm{C}_{3}(\mathbf{C})$ paramétrés par les orbites $\{f g, g f\},\{e f, e g\},\{f e, g e\}$ de $\Sigma_{0}$ dans $\mathcal{O}_{3}$ respectivement et $\mathrm{B}_{0}$, $\mathrm{B}_{1}, \mathrm{~B}_{2}$ les sommets de type 1 paramétrés par les orbites $\{b b, e f, d d, f e\}$, $\{g e, h b, g f, h d\},\{e g, d h, f g, b h\}$ de $\Sigma_{1}$. Les coordonnées homogènes de ces points sont

$$
\begin{array}{ll}
\mathrm{A}_{0}^{\prime}=\left(\alpha_{1}, 0,1\right), & \mathrm{B}_{0}=(1,0,1), \\
\mathrm{A}_{1}^{\prime}=\left(\frac{\alpha_{0}+\alpha_{1}}{2}, \frac{i\left(\alpha_{1}-\alpha_{0}\right)}{2}, 1\right), & \mathrm{B}_{1}=\left(\frac{\beta_{0}+1}{2}, \frac{i\left(1-\beta_{0}\right)}{2}, 1\right), \\
\mathrm{A}_{2}^{\prime}=\left(\frac{\alpha_{0}+\alpha_{1}}{2}, \frac{i\left(\alpha_{0}-\alpha_{1}\right)}{2}, 1\right), & \mathrm{B}_{2}=\left(\frac{\beta_{0}+1}{2}, \frac{i\left(\beta_{0}-1\right)}{2}, 1\right) .
\end{array}
$$

La restriction à la courbe $\mathrm{C}_{3}$ du fibré $\mathcal{O}(1)$ sur le plan projectif est un fibré inversible de degré 3 ; les coordonnées homogènes $\underline{x}, \underline{y}, \underline{t}$ peuvent être considérées comme des sections de ce fibré dont les diviseurs sont

(25) $\operatorname{div}(\underline{x})=2 \mathrm{~A}_{0}+\mathrm{D}_{0}, \operatorname{div}(\underline{y})=\mathrm{A}_{0}+\mathrm{A}_{0}^{\prime}+\mathrm{B}_{0}, \operatorname{div}(\underline{t})=\mathrm{D}_{0}+\mathrm{D}_{1}+\mathrm{D}_{2}$.

Par ailleurs, on a

$$
\begin{aligned}
\operatorname{div}\left(\underline{x}-\frac{\alpha_{2}}{2} \underline{t}\right) & =\mathrm{A}_{1}+\mathrm{A}_{2}+\mathrm{D}_{0}, & \operatorname{div}(\underline{x}-\delta \underline{t}) & =3 \mathrm{D}_{0}, \\
\operatorname{div}\left(\underline{x}-\frac{\beta_{0}+1}{2} \underline{t}\right) & =\mathrm{B}_{1}+\mathrm{B}_{2}+\mathrm{D}_{0}, & \operatorname{div}(\underline{x}-\underline{t}) & =2 \mathrm{~B}_{0}+\mathrm{D}_{0}, \\
\operatorname{div}\left(\underline{x}-\frac{\alpha_{0}+\alpha_{1}}{2} \underline{t}\right) & =\mathrm{A}_{1}^{\prime}+\mathrm{A}_{2}^{\prime}+\mathrm{D}_{0}, & \operatorname{div}\left(\underline{x}-\alpha_{1} \underline{t}\right) & =2 \mathrm{~A}_{0}^{\prime}+\mathrm{D}_{0} .
\end{aligned}
$$

Nous allons maintenant exprimer $\mathrm{X}$ en termes des fonctions rationnelles $x=\underline{x} / \underline{t}$ et $y=\underline{y} / \underline{t}$. Vu les relations précédentes, on a

$$
\begin{aligned}
\operatorname{div}(\mathrm{X}) & =\operatorname{div}(\underline{x})+2 \operatorname{div}\left(\underline{x}-\frac{\alpha_{2}}{2} \underline{t}\right)-2 \operatorname{div}(\underline{t})-\operatorname{div}(\underline{x}-\delta \underline{t}) \\
& =\operatorname{div}(x)+2 \operatorname{div}\left(x-\frac{\alpha_{2}}{2}\right)-\operatorname{div}(x-\delta) .
\end{aligned}
$$


Il existe par suite une constante $\mu$ telle que

$$
\mathrm{X}=\frac{x\left(x-\frac{\alpha_{2}}{2}\right)^{2}}{\mu(x-\delta)} .
$$

On démontre de même, par comparaison des diviseurs, que l'on a

$$
\mathrm{X}-1=\frac{(x-1)\left(x-\frac{\beta_{0}+1}{2}\right)^{2}}{\mu(x-\delta)}, \quad \mathrm{X}-\frac{3}{2}=\frac{\left(x-\alpha_{1}\right)\left(x-\frac{\alpha_{0}+\alpha_{1}}{2}\right)^{2}}{\mu(x-\delta)} .
$$

La première de ces égalités se traduit par les relations

$$
\beta_{0}=\alpha_{2}-2, \quad \mu=\frac{3-2 \alpha_{2}}{4}, \quad \delta=\frac{\left(\alpha_{2}-1\right)^{2}}{2 \alpha_{2}-3} .
$$

En substituant ces valeurs de $\mu$ et $\delta$ dans la seconde, on obtient

$$
\alpha_{0}+2 \alpha_{1}=\alpha_{2}, \alpha_{1}\left(2 \alpha_{0}+\alpha_{1}\right)=\frac{3}{2}\left(2 \alpha_{2}-3\right), \alpha_{1}\left(\alpha_{0}+\alpha_{1}\right)^{2}=\frac{3}{2}\left(\alpha_{2}-1\right)^{2} .
$$

Des deux premières égalités de (33), on tire $\alpha_{1}\left(2 \alpha_{2}-3 \alpha_{1}\right)=\frac{3}{2}\left(2 \alpha_{2}-3\right)$, c'est-à-dire

$$
\alpha_{2}=\frac{3\left(2 \alpha_{1}^{2}-3\right)}{2\left(2 \alpha_{1}-3\right)}
$$

La dernière relation de (33) peut alors se récrire $\alpha_{1}\left(\alpha_{2}-\alpha_{1}\right)^{2}=\frac{3}{2}\left(\alpha_{2}-\right.$ $1)^{2}$, c'est-à-dire $\alpha_{2}^{2}\left(2 \alpha_{1}-3\right)-2 \alpha_{2}\left(2 \alpha_{1}^{2}-3\right)+\left(2 \alpha_{1}^{3}-3\right)=0$, ou encore, en remplaçant $\alpha_{2}$ par son expression (34) en fonction de $\alpha_{1}$,

$$
\begin{gathered}
3\left(2 \alpha_{1}^{2}-3\right)^{2}=3\left(2 \alpha_{1}-3\right)\left(2 \alpha_{1}^{3}-3\right), \\
\text { i.e. } \quad 4 \alpha_{1}^{4}-24 \alpha_{1}^{3}+36 \alpha_{1}^{2}-24 \alpha_{1}+9=0 .
\end{gathered}
$$

De (34) et (35), on déduit que l'on a

$$
\alpha_{1}=\frac{\alpha_{2}\left(\alpha_{2}-2\right)}{2 \alpha_{2}-3} \text {. }
$$

Il est commode pour comparer ces résultats à ceux de 5.2 d'exprimer tous les nombres précédents en fonction de $\alpha=\frac{\alpha_{2}-3}{2 \alpha_{2}-3}$. On a

$$
\begin{aligned}
& \alpha_{2}=\frac{3(1-\alpha)}{1-2 \alpha}, \quad \alpha_{1}=\frac{1-\alpha^{2}}{1-2 \alpha}, \quad \alpha_{0}=1-\alpha, \\
& \beta_{0}=\frac{1+\alpha}{1-2 \alpha}, \quad \mu=-\frac{3}{4(1-2 \alpha)}, \quad \delta=\frac{(2-\alpha)^{2}}{3(1-2 \alpha)} .
\end{aligned}
$$

En remplaçant ces expressions dans la seconde relation (33), on obtient l'équation algébrique satisfaite par $\alpha$

$$
2 \alpha^{4}-4 \alpha^{3}-2 \alpha+1=0 .
$$

Cette équation a deux racines réelles dont l'une est $<1$ et l'autre $>1$. Comme on sait que $\alpha_{0}$ est positif, $\alpha$ est la plus petite de ces deux racines. 
De la relation $\delta=\left(\alpha_{0}+2 \alpha_{1}+2 \alpha_{2}-3 \gamma\right) / 6$ énoncée au début de ce numéro, on déduit la valeur de $\gamma$ :

$$
\gamma=\frac{1+\alpha}{3}
$$

Si un point complexe de $\mathrm{C}_{3} \cap \mathrm{U}$ a pour coordonnées $(x, y)$, on a $f(x+i y)=$ $-\mathrm{X}^{2}(2 \mathrm{X}-3)$ où $\mathrm{X}=\frac{x\left(x-\frac{\alpha_{2}}{2}\right)^{2}}{\mu(x-\delta)}$; lorsqu'un tel point tend vers le point à l'infini de coordonnées homogènes $(1,-i, 0), x$ tend vers l'infini, $\mathrm{X}$ est équivalent à $\frac{1}{\mu}, x+i y$ à $2 x$ et $f(x+i y)$ à $\lambda(2 x)^{6}$; on a donc $2^{6} \lambda=-\frac{2}{\mu^{3}}$, d'où

$$
\lambda=\frac{2(1-2 \alpha)^{3}}{27} .
$$

Nous avons ainsi déterminé tous les coefficients de la fonction de Belyi $f$.

Remarque 2. Notons $\mathrm{B}_{0}^{\prime}, \mathrm{B}_{0}^{\prime \prime}, \mathrm{B}_{1}^{\prime}, \mathrm{B}_{1}^{\prime \prime}, \mathrm{B}_{2}^{\prime}, \mathrm{B}_{2}^{\prime \prime}$, les sommets de type 1 de $\mathrm{C}_{3}(\mathbf{C})$ paramétrés par les orbites $\{a c\},\{c a\},\{j a\},\{j c\},\{c j\},\{a j\}$ de $\Sigma_{1}$ dans $\mathcal{O}_{3}$ respectivement (cf. figure 11). Les coordonnées homogènes de ces points sont

$$
\begin{array}{ll}
\mathrm{B}_{0}^{\prime}=\left(\frac{\beta_{1}+\beta_{2}}{2}, \frac{i\left(\beta_{1}-\beta_{2}\right)}{2}, 1\right), & \mathrm{B}_{0}^{\prime \prime}=\left(\frac{\beta_{1}+\beta_{2}}{2}, \frac{i\left(\beta_{2}-\beta_{1}\right)}{2}, 1\right), \\
\mathrm{B}_{1}^{\prime}=\left(\frac{\beta_{2}+\beta_{3}}{2}, \frac{i\left(\beta_{2}-\beta_{3}\right)}{2}, 1\right), & \mathrm{B}_{1}^{\prime \prime}=\left(\frac{\beta_{1}+\beta_{3}}{2}, \frac{i\left(\beta_{1}-\beta_{3}\right)}{2}, 1\right), \\
\mathrm{B}_{2}^{\prime}=\left(\frac{\beta_{1}+\beta_{3}}{2}, \frac{i\left(\beta_{3}-\beta_{1}\right)}{2}, 1\right), & \mathrm{B}_{2}^{\prime \prime}=\left(\frac{\beta_{2}+\beta_{3}}{2}, \frac{i\left(\beta_{3}-\beta_{2}\right)}{2}, 1\right) .
\end{array}
$$

On a

(43) $\operatorname{div}\left(\mathrm{X}+\frac{1}{2}\right)=\mathrm{B}_{0}^{\prime}+\mathrm{B}_{0}^{\prime \prime}+\mathrm{B}_{1}^{\prime}+\mathrm{B}_{1}^{\prime \prime}+\mathrm{B}_{2}^{\prime}+\mathrm{B}_{2}^{\prime \prime}-2 \mathrm{D}_{0}-2 \mathrm{D}_{1}-2 \mathrm{D}_{2}$,

$$
\begin{gathered}
\operatorname{div}\left(x-\frac{\beta_{1}+\beta_{2}}{2}\right)=\mathrm{B}_{0}^{\prime}+\mathrm{B}_{0}^{\prime \prime}-\mathrm{D}_{1}-\mathrm{D}_{2} \\
\operatorname{div}\left(x-\frac{\beta_{2}+\beta_{3}}{2}\right)=\mathrm{B}_{1}^{\prime}+\mathrm{B}_{2}^{\prime \prime}-\mathrm{D}_{1}-\mathrm{D}_{2} \\
\operatorname{div}\left(x-\frac{\beta_{1}+\beta_{3}}{2}\right)=\mathrm{B}_{1}^{\prime \prime}+\mathrm{B}_{2}^{\prime}-\mathrm{D}_{1}-\mathrm{D}_{2} \\
\operatorname{div}(x-\delta)=2 \mathrm{D}_{0}-\mathrm{D}_{1}-\mathrm{D}_{2}
\end{gathered}
$$

On en déduit que l'on a

$$
\mathrm{X}+\frac{1}{2}=\frac{\left(x-\frac{\beta_{1}+\beta_{2}}{2}\right)\left(x-\frac{\beta_{2}+\beta_{3}}{2}\right)\left(x-\frac{\beta_{1}+\beta_{3}}{2}\right)}{\mu(x-\delta)} .
$$

Si l'on note $\mathrm{R}(x)=x^{3}-s_{1} x^{2}+s_{2} x-s_{3}$ le polynôme $\left(x-\beta_{1}\right)\left(x-\beta_{2}\right)\left(x-\beta_{3}\right)$, la relation (48) s'écrit

$$
x\left(x-\frac{\alpha_{2}}{2}\right)^{2}+\frac{\mu}{2}(x-\delta)=\frac{-1}{8} \mathrm{R}\left(s_{1}-2 x\right)
$$


d'où l'on déduit $s_{1}=\alpha_{2}, s_{2}=2 \mu, s_{3}=2 \mu\left(\alpha_{2}-2 \delta\right)$, c'est-à-dire

$$
s_{1}=\frac{3(1-\alpha)}{1-2 \alpha}, \quad s_{2}=-\frac{3}{2(1-2 \alpha)}, \quad s_{3}=-\frac{\alpha+1}{2(1-2 \alpha)} .
$$

On retrouve ainsi le facteur de degré 3 de l'expression $(7)$ de $f-1$.

Remarque 3. Le calcul de $f$ présenté dans ce numéro explique la forme très simple que prennent les coefficients des différents facteurs de $f$ et $f-1$ dans les formules (6) et (7), lorsqu'on les exprime en fonction de $\alpha$.

Remarque 4. Identifions comme-ci-dessus la courbe $\mathrm{C}^{\prime}$ à la droite projective. Les points $\infty, 0,1$ et $\frac{3}{2}$ de $\mathrm{C}^{\prime}$ ont chacun un unique antécédent par $h$ dans $\mathrm{C}$. Notons $\mathrm{O}$ l'antécédent de $\infty$. La courbe $\mathrm{C}$, munie du point rationnel $\mathrm{O}$ est une courbe elliptique; ses trois points d'ordre deux sont les antécédents de 0,1 et $\frac{3}{2}$ par $h$. Il existe une fonction rationnelle sur C, unique à multiplication près par une constante, ayant pour seul pôle un pôle triple en l'origine et s'annulant en chacun des points d'ordre 2. Sa composée avec le morphisme canonique $\pi: \mathrm{C}_{3} \rightarrow \mathrm{C}$ est une fonction rationnelle $\mathrm{Y}$ sur $\mathrm{C}_{3}$ dont le diviseur est $\mathrm{A}_{0}+\mathrm{A}_{1}+\mathrm{A}_{2}+\mathrm{A}_{0}^{\prime}+\mathrm{A}_{1}^{\prime}+\mathrm{A}_{2}^{\prime}+$ $\mathrm{B}_{0}+\mathrm{B}_{1}+\mathrm{B}_{2}-3 \mathrm{D}_{0}-3 \mathrm{D}_{1}+\mathrm{D}_{2}$. Vu les relations (25) à (28), on peut la normaliser en prenant

$$
\mathrm{Y}=y \frac{\left(x-\frac{\alpha_{2}}{2}\right)\left(x-\frac{\beta_{0}+1}{2}\right)\left(x-\frac{\alpha_{0}+\alpha_{1}}{2}\right)}{x-\delta}
$$

et l'on a alors

$$
\mathrm{Y}^{2}=-\mu^{3} \mathrm{X}(\mathrm{X}-1)\left(\mathrm{X}-\frac{3}{2}\right)
$$

Cette équation fournit un modèle de Weierstrass affine de $\mathrm{C}$, les expressions de $\mathrm{X}$ et $\mathrm{Y}$ en fonction de $x$ et $y$ décrivant le morphisme $\pi: \mathrm{C}_{3} \rightarrow \mathrm{C}$ dans ce modèle.

Remarque 5. L'automorphisme d'ordre 2 de $\mathrm{C}_{3}$ considéré dans la remarque 1 est $(x, y) \mapsto(x,-y)$.

Remarque 6. L'automorphisme d'ordre 3 de la complexifiée de $\mathrm{C}_{3}$ associé à la permutation $\tau$ définie dans la formule (15) est

$$
(x, y) \mapsto\left(\frac{-x+i y+\alpha_{2}}{2}, \frac{3 i x-y-i \alpha_{2}}{2}\right) .
$$

\section{Composantes irréductibles de $\Gamma_{(\mathrm{C})}$ : degré en genre 0 , classe dans $\operatorname{Pic}\left(\mathrm{X} \times \mathrm{X}^{*}\right)$}

Après un $\S 7.1$ consacré à des rappels sur les diviseurs, le groupe de Picard et ses propriétés de fonctorialité, nous reprendrons les notations des chapitres précédents. Toute composante irréductible $\mathrm{C}$ de $\Gamma_{(\mathbf{C})}$ est un diviseur de $\mathrm{X} \times \mathrm{X}^{*}$. Nous notons $[\mathrm{C}]$ sa classe dans le groupe de Picard $\operatorname{Pic}\left(\mathrm{X} \times \mathrm{X}^{*}\right)$ 
$\mathrm{Au} \S 7.2$, nous considérons le cas où la courbe $\mathrm{X}$ est connexe et de genre 0 , i.e. isomorphe à $\mathbf{P}^{1}$. Dans ce cas $\operatorname{Pic}\left(\mathrm{X} \times \mathrm{X}^{*}\right)$ s'identifie à $\mathbf{Z} \times \mathbf{Z}$ et $[\mathrm{C}]$ à un couple d'entiers appelé le bidegré de $\mathrm{C}$; nous l'exprimons en termes de l'orbite $\mathcal{O}$ de $\left\langle\Sigma_{0}, \Sigma_{1}\right\rangle$ dans $\mathrm{E} \times \mathrm{E}$ associée à $\mathrm{C}$. Le choix d'un isomorphisme $\mathrm{X} \approx \mathbf{P}^{1}$ permet de considérer la trace de $\mathrm{C}$ sur le plan affine $\mathbf{A}^{1} \times \mathbf{A}^{1}$. C'est une courbe affine dont nous calculons le degré; il dépend du point de $\mathrm{X}$ qui correspond au point à l'infini de $\mathbf{P}^{1}$ par l'isomorphisme choisi.

$\mathrm{Au} \S 7.3$, nous considérons le cas où $\mathrm{X}$ est connexe de genre quelconque. Dans ce cas, la structure du groupe de Picard de $\mathrm{X} \times \mathrm{X}^{*}$ est plus compliquée : on dispose d'une suite exacte canonique

$$
0 \rightarrow \operatorname{Pic}(\mathrm{X}) \times \operatorname{Pic}\left(\mathrm{X}^{*}\right) \rightarrow \operatorname{Pic}\left(\mathrm{X} \times \mathrm{X}^{*}\right) \rightarrow \operatorname{Hom}\left(\operatorname{Jac}(\mathrm{X}), \operatorname{Jac}\left(\mathrm{X}^{*}\right)\right) \rightarrow 0,
$$

où $\mathrm{Jac}(\mathrm{X})$ désigne la variété Jacobienne de $\mathrm{X}$; les flèches sont précisées au $\S$ 7.1.6. Nous montrons par un exemple que les images des classes [C] des composantes irréductibles $\mathrm{C}$ de $\Gamma_{(\mathbf{C})}$ dans $\operatorname{Hom}\left(\operatorname{Jac}(\mathrm{X}), \operatorname{Jac}\left(\mathrm{X}^{*}\right)\right)$ peuvent être non nulles bien que leur somme soit 0 .

\subsection{Rappels sur le groupe de Picard.}

Dans ce paragraphe, $\mathrm{S}$ désigne une variété algébrique complexe irréductible non singulière (i.e. un schéma de type fini sur $\mathbf{C}$, irréductible et lisse).

\subsubsection{Diviseurs (de Weil).}

Un diviseur irréductible de $\mathrm{S}$ est par définition un sous-schéma intègre $\mathrm{Z}$ de codimension 1 de $\mathrm{S}$. La donnée d'un tel sous-schéma équivaut à celle de son point générique, c'est-à-dire d'un point $z$ de du schéma $S$ tel que $\mathcal{O}_{\mathrm{S}, z}$ soit de dimension de Krull 1 ; l'anneau $\mathcal{O}_{\mathrm{S}, z}$ est alors un anneau de valuation discrète.

On appelle diviseur (ou plus précisément diviseur de Weil) de $\mathrm{S}$ tout élément $\mathrm{D}=\sum_{\mathrm{Z}} m_{\mathrm{Z}} \mathrm{Z}$ du groupe abélien libre construit sur l'ensemble des diviseurs irréductibles de $\mathrm{S}$. Le support de $\mathrm{D}$ est la réunion des $\mathrm{Z}$ tels que $m_{Z} \neq 0$. On dit que le diviseur D est effectif si tous les entiers $m_{Z}$ sont $\geq 0$. On dit que $\mathrm{D}$ est supérieur à un diviseur $\mathrm{D}^{\prime}$ si $\mathrm{D}-\mathrm{D}^{\prime}$ est effectif.

Soit $\mathrm{Z}$ un diviseur irréductible de $\mathrm{S}$ et $z$ son point générique. La valuation de l'anneau local $\mathcal{O}_{\mathrm{S}, z}$ se prolonge de manière unique en une valuation discrète normée $v_{\mathrm{Z}}$ sur le corps des fonctions rationnelles sur $\mathrm{S}$.

Si $f$ est une fonction rationnelle non nulle sur S, l'ensemble des diviseurs irréductibles $\mathrm{Z}$ tels que $v_{\mathrm{Z}}(f) \neq 0$ est fini. Le diviseur $\sum_{\mathrm{Z}} v_{\mathrm{Z}}(f) \mathrm{Z}$ se note $\operatorname{div}(f)$ et s'appelle le diviseur de $f$. Les diviseurs de cette forme sont dits principaux.

L'ensemble des diviseurs principaux est un sous-groupe du groupe des diviseurs de S. Le groupe quotient s'appelle le groupe des classes de diviseurs de $\mathrm{S}$. 


\subsubsection{Diviseurs de Cartier.}

Soit $\mathcal{K}$ le faisceau constant sur $\mathrm{S}$ des fonctions rationnelles. Un diviseur de Cartier de $\mathrm{S}$ est par définition un sous- $\mathcal{O}_{\mathrm{S}}$-module localement libre de rang 1 de $\mathcal{K}$. Il est dit effectif s'il contient $\mathcal{O}_{\mathrm{S}}$, principal s'il est libre.

L'ensemble des diviseurs de Cartier de $\mathrm{S}$ est muni d'une structure de groupe abélien dont la loi de composition est $(\mathcal{I}, \mathcal{J}) \mapsto \mathcal{I} \mathcal{J}$. Les diviseurs de Cartier principaux en forment un sous-groupe; le groupe quotient s'appelle le groupe des classes de diviseurs de Cartier de $\mathrm{S}$.

Soit $\mathrm{D}=\sum_{\mathrm{Z}} m_{\mathrm{Z}} \mathrm{Z}$ un diviseur de Weil de $\mathrm{S}$. Notons $\mathcal{O}_{\mathrm{S}}(\mathrm{D})$ le sous- $\mathcal{O}_{\mathrm{S}^{-}}$ module de $\mathcal{K}$ défini comme suit : ses sections sur un ouvert non vide $U$ de $\mathrm{S}$ sont les fonctions rationnelles $f$ sur $\mathrm{S}$ dont le diviseur est supérieur à $-\mathrm{D}$ sur $\mathrm{U}$, i.e. telles que $v_{\mathrm{Z}}(f)+m_{\mathrm{Z}} \geq 0$ pour tout diviseur irréductible $\mathrm{Z}$ qui rencontre $\mathrm{U}$. Le fait que $\mathrm{S}$ soit lisse implique que $\mathcal{O}_{\mathrm{S}}(\mathrm{D})$ est un $\mathcal{O}_{\mathrm{S}}$-module localement libre de rang 1 , i.e. un diviseur de Cartier de $\mathrm{S}$.

L'application $\mathrm{D} \mapsto \mathcal{O}_{\mathrm{S}}(\mathrm{D})$ est un isomorphisme du groupe des diviseurs de Weil de S sur celui des diviseurs de Cartier de S, par lequel les diviseurs effectifs (resp. principaux) se correspondent. On en déduit par passage au quotient un isomorphisme entre les groupes des classes de diviseurs de Weil et de Cartier.

\subsubsection{Le groupe de Picard.}

On appelle module inversible sur $\mathrm{S}$ un $\mathcal{O}_{\mathrm{S}}$-module localement libre de rang 1. L'ensemble des classes d'isomorphisme de modules inversibles sur $\mathrm{S}$, muni de la loi de composition déduite du produit tensoriel, est un groupe abélien appelé le groupe de Picard de $\mathrm{S}$ et noté $\mathrm{Pic}(\mathrm{S})$.

L'application qui à un diviseur de Cartier $\mathcal{I}$ sur $\mathrm{S}$ associe la classe d'isomorphisme du $\mathcal{O}_{\mathrm{S}}$-module $\mathcal{I}$ est un isomorphisme du groupe des classes de diviseurs de Cartier de $\mathrm{S}$ sur $\operatorname{Pic}(\mathrm{S})$.

Il en résulte, d'après 7.1.2, que l'application qui à un diviseur de Weil $\mathrm{D}$ sur $\mathrm{S}$ associe la classe d'isomorphisme du $\mathcal{O}_{\mathrm{S}}$-module $\mathcal{O}_{\mathrm{S}}(\mathrm{D})$, souvent notée [D], est un isomorphisme du groupe des classes de diviseurs de Weil de $\mathrm{S}$ sur $\operatorname{Pic}(\mathrm{S})$.

\subsubsection{Propriétés de fonctorialité du groupe de Picard.}

Soient $\mathrm{S}$ et $\mathrm{T}$ deux variétés algébriques complexes irréductibles non singulières et $u$ un morphisme (de $\mathbf{C}$-schémas) de $\mathrm{S}$ dans $\mathrm{T}$.

On définit un homomorphisme de groupes $u^{*}: \operatorname{Pic}(\mathrm{T}) \rightarrow \mathrm{Pic}(\mathrm{S})$ en associant à la classe d'un $\mathcal{O}_{\mathrm{T}}$-module inversible $\mathcal{L}$ celle du $\mathcal{O}_{\mathrm{S}}$-module inversible déduit de $\mathcal{L}$ par le changement de base $u$.

7.1.5. Le groupe de Picard d'une courbe projective irréductible.

Soit $\mathrm{S}$ une courbe projective lisse et irréductible. Un diviseur de S n'est alors autre qu'une combinaison linéaire $\mathrm{D}=\sum_{s} m_{s} s$ (à support fini et à coefficients dans $\mathbf{Z}$ ) des points fermés de S. L'entier $\sum_{s} m_{s}$ s'appelle le degré 
du diviseur $\mathrm{D}$ et se note $\operatorname{deg}(\mathrm{D})$. Tout diviseur principal est de degré 0 . Le groupe des classes de diviseurs de degré 0 de $\mathrm{S}$ s'identifie canoniquement au groupe des points complexes de la jacobienne $\operatorname{Jac}(\mathrm{S})$ de $\mathrm{S}$.

7.1.6. Le groupe de Picard du produit de deux courbes projectives irréductibles.

Soient $\mathrm{S}$ et $\mathrm{T}$ deux courbes projectives lisses et irréductibles sur C. Notons $\operatorname{Jac}(\mathrm{S})$ et $\operatorname{Jac}(\mathrm{T})$ leurs jacobiennes. Notons $p$ et $q$ les projections de $\mathrm{S} \times \mathrm{T}$ sur $\mathrm{S}$ et $\mathrm{T}$ respectivement. Si $s \in \mathrm{S}$, notons $a_{s}$ le morphisme $t \mapsto(s, t)$ de $\mathrm{T}$ dans $\mathrm{S} \times \mathrm{T}$.

D'après [1], chap. $11, \S 5$, th. 5.1 , il existe une suite exacte

$$
0 \rightarrow \operatorname{Pic}(\mathrm{S}) \times \operatorname{Pic}(\mathrm{T}) \stackrel{\alpha}{\longrightarrow} \operatorname{Pic}(\mathrm{S} \times \mathrm{T}) \stackrel{\beta}{\longrightarrow} \operatorname{Hom}(\operatorname{Jac}(\mathrm{S}), \operatorname{Jac}(\mathrm{T})) \rightarrow 0,
$$

où $\alpha(\xi, \eta)=p^{*}(\xi)+q^{*}(\eta)$ et $\beta$ est caractérisé par la propriété suivante : si $\xi$ est un élément de $\operatorname{Pic}(\mathrm{S} \times \mathrm{T})$ et $u$ son image par $\beta$, l'application $\underline{u}$ : $\operatorname{Jac}(\mathrm{S})(\mathbf{C}) \rightarrow \operatorname{Jac}(\mathrm{T})(\mathbf{C})$ déduite de $u$ par passage aux points complexes applique la classe d'un diviseur $\sum n_{i} s_{i}$ de degré 0 sur $\mathrm{S}$ sur $\sum n_{i} a_{s_{i}}^{*}(\xi)$.

\subsection{Degré des composantes irréductibles de $\Gamma_{(C)}$ lorsque $X=P^{1}$.}

Nous reprenons maintenant les notations du $\S 1$. Dans ce paragraphe, nous supposons de plus que la courbe $\mathrm{X}$ est connexe et de genre 0 . Les groupes $\mathrm{Pic}(\mathrm{X})$ et $\mathrm{Pic}\left(\mathrm{X}^{*}\right)$ sont alors canoniquement isomorphes à $\mathbf{Z}$ et le groupe $\operatorname{Pic}\left(\mathrm{X} \times \mathrm{X}^{*}\right)$ à $\operatorname{Pic}(\mathrm{X}) \times \operatorname{Pic}\left(\mathrm{X}^{*}\right)$, donc à $\mathbf{Z} \times \mathbf{Z}$. La classe d'un diviseur $\mathrm{D}$ de $\mathrm{X} \times \mathrm{X}^{*}$ peut ainsi être représentée par un couple $\left(d^{\prime}, d^{\prime \prime}\right)$ d'entiers relatifs, que l'on appelle le bidegré de D.

\subsubsection{Bidegré d'une composante irréductible $\mathrm{C}$ de $\Gamma_{(\mathbf{C})}$.}

Soit $\mathrm{C}$ une composante irréductible de $\Gamma_{(\mathbf{C})}$. Notons $\mathcal{O}$ l'orbite de $\left\langle\Sigma_{0}, \Sigma_{1}\right\rangle$ dans $\mathrm{E} \times \mathrm{E}$ qui lui est associée par la bijection (1) de [2]§2.1.1. Par le plongement canonique de $\Gamma_{(\mathbf{C})}$ dans $\mathrm{X} \times \mathrm{X}^{*}, \mathrm{C}$ s'identifie à un diviseur irréductible de $\mathrm{X} \times \mathrm{X}^{*}$.

Proposition 7.1. Le bidegré de $\mathrm{C}$ est $\left(d_{\mathrm{C}}, d_{\mathrm{C}}\right)$, où $d_{\mathrm{C}}=\operatorname{Card}(\mathcal{O}) / n$.

Soit $\left(d^{\prime}, d^{\prime \prime}\right)$ ce bidegré. L'entier $d^{\prime \prime}$ est le degré de $a_{x}^{*}(\mathcal{O}(\mathrm{C}))$, où $x$ est un point fermé quelconque de $\mathrm{X}$ et $a_{x}: \mathrm{X}^{*} \rightarrow \mathrm{X} \times \mathrm{X}^{*}$ le morphisme $y \mapsto(x, y)$; c'est donc le degré $d_{\mathrm{C}}$ du morphisme $\pi_{\mathrm{C}}: \mathrm{C} \rightarrow \mathrm{X}$ déduit par restriction de la première projection. Celui-ci a été déterminé [2] $\$ 2.4 .2$ : il est égal à $\operatorname{Card}(\mathcal{O}) / n$. De même, $d^{\prime}$ est le degré du morphisme $\pi_{\mathrm{C}}^{\prime}: \mathrm{C} \rightarrow \mathrm{X}^{*}$ déduit par restriction de la deuxième projection; il est aussi égal à $d_{\mathrm{C}}$ (loc.cit., remarque 2).

7.2.2. Définition du degré d'une composante irréductible de $\Gamma_{(\mathbf{C})}$, lorsque $\mathrm{X}=\mathbf{P}^{1}$.

Dans la suite de ce paragraphe, nous supposons qu'un isomorphisme de $\mathrm{X}$ avec la droite projective a été choisi, et nous identifions $\mathrm{X}$ à $\mathbf{P}^{1}$ par cet 
isomorphisme. Le revêtement ramifié $f: \mathrm{X} \rightarrow \mathbf{P}^{1}$ est alors une fonction de Belyi de la forme $\mathrm{P} / \mathrm{Q}$, où $\mathrm{P}$ et $\mathrm{Q}$ sont des polynômes premiers entre eux, avec $\mathrm{Q}$ unitaire; son degré $n$ est égal à $\sup (\operatorname{deg}(\mathrm{P}), \operatorname{deg}(\mathrm{Q}))$.

Soit U l'ouvert $\prod_{\mathbf{C} / \mathbf{R}} \mathbf{A}^{1}$ de $\prod_{\mathbf{C} / \mathbf{R}} \mathbf{P}^{1}$. Il s'identifie canoniquement à un plan affine défini sur $\mathbf{R}$, muni de coordonnées $x, y$. Lorsqu'on identifie le complexifié de $\prod_{\mathbf{C} / \mathbf{R}} \mathbf{P}^{1}$ à $\mathbf{P}^{1} \times \mathbf{P}^{1}$, le complexifié de $\mathbf{U}$ s'identifie à $\mathbf{A}^{1} \times \mathbf{A}^{1}$, qui est un plan affine défini sur $\mathbf{C}$, muni de coordonnées $z, z^{\prime}$. On prendra garde que ces coordonnées ne coïncident pas avec $x, y$, mais que l'on a $z=x+i y$, $z^{\prime}=x-i y$.

La trace de $\Gamma$ sur $U$ est une courbe affine dont une équation, dans le système de coordonnées $(x, y)$, s'obtient en écrivant que le numérateur de la partie imaginaire de $f(x+i y)$ est nul, c'est-à-dire

$$
\frac{1}{2 i}(\mathrm{P}(x+i y) \overline{\mathrm{Q}(x+i y)}-\mathrm{Q}(x+i y) \overline{\mathrm{P}(x+i y)})=0 .
$$

Une équation de la trace de $\Gamma_{(\mathbf{C})}$ sur $\mathrm{U}_{(\mathbf{C})} \approx \mathbf{A}^{1} \times \mathbf{A}^{1}$, dans le système de coordonnées $\left(z, z^{\prime}\right)$ est $\mathrm{P}(z) \overline{\mathrm{Q}}\left(z^{\prime}\right)-\mathrm{Q}(z) \overline{\mathrm{P}}\left(z^{\prime}\right)=0$, où $\overline{\mathrm{P}}$ et $\overline{\mathrm{Q}}$ sont les conjugués des polynômes $\mathrm{P}$ et $\mathrm{Q}$.

Les traces sur $\mathbf{A}^{1} \times \mathbf{A}^{1}$ des différentes composantes irréductibles de $\Gamma_{(\mathbf{C})}$ ont pour équations dans les systèmes de coordonnées $(x, y)$ et $\left(z, z^{\prime}\right)$ les facteurs irréductibles (sur $\mathbf{C}$ ) des équations précédentes. Leurs adhérences dans $\mathbf{P}^{2}$ sont des courbes projectives planes irréductibles (en général singulières), dont les équations, dans les systèmes de coordonnées homogènes associés $(\underline{x}, \underline{y}, \underline{t})$ et $\left(\underline{z}, \underline{z}^{\prime}, \underline{t}\right)$, sont les homogénéisées des équations affines précédentes.

Soit $\mathrm{C}$ une composante irréductible de $\Gamma_{(\mathbf{C})}$. Le degré de l'équation affine de $\mathrm{C} \cap\left(\mathbf{A}^{1} \times \mathbf{A}^{1}\right)$ (dans le système de coordonnées $(x, y)$ ou $\left(z, z^{\prime}\right)$, cela revient au même) sera appelé par abus le degré de $\mathrm{C}:$ c'est le degré de la courbe projective plane $\widehat{\mathrm{C}}$, adhérence de $\mathrm{C} \cap\left(\mathbf{A}^{1} \times \mathbf{A}^{1}\right)$ dans $\mathbf{P}^{2}$.

7.2.3. Calcul du degré d'une composante irréductible de $\Gamma_{(\mathbf{C})}$, lorsque $\mathrm{X}=$ $\mathbf{P}^{1}$.

Reprenons les notations du numéro précédent. Soit $\mathrm{C}$ une composante irréductible de $\Gamma_{(\mathbf{C})}$. Notons $m(\mathrm{C})$ la multiplicité de $\mathrm{C}$ en le point $(\infty, \infty)$ de $\mathbf{P}^{1} \times \mathbf{P}^{1}$ (avec par convention $m(\mathrm{C})=0$ si $\mathrm{C}$ ne passe pas par ce point, ce qui est par exemple le cas lorsque $\mathrm{C}$ n'est pas réelle).

Proposition 7.2. Le degré de $\mathrm{C}$ est $2 d_{\mathrm{C}}-m(\mathrm{C})$

Pour le démontrer, nous comparerons la classe $[\mathrm{C}]$ de $\mathrm{C}$ dans $\operatorname{Pic}\left(\mathbf{P}^{1} \times \mathbf{P}^{1}\right)$ à la classe $[\widehat{\mathrm{C}}]$ de $\widehat{\mathrm{C}}$ dans $\operatorname{Pic}\left(\mathbf{P}^{2}\right)$. Rappelons que l'on identifie $\operatorname{Pic}\left(\mathbf{P}^{1} \times \mathbf{P}^{1}\right)$ à $\mathbf{Z}^{2}$ (les classes des diviseurs formés par des droites verticales et horizontales s'identifiant respectivement à $(1,0)$ et $(0,1))$ et $\operatorname{Pic}\left(\mathbf{P}^{2}\right)$ à $\mathbf{Z}$ (la classe du 
diviseur formé par une droite de $\mathbf{P}^{2}$ s'identifiant à 1). Ceci fait, [C] s'identifie à $\left(d_{\mathrm{C}}, d_{\mathrm{C}}\right)$ d'après la prop. 7.1 et $[\widehat{\mathrm{C}}]$ au degré $\delta$ que nous cherchons à déterminer.

Soit $u: \mathrm{S} \rightarrow \mathbf{P}^{1} \times \mathbf{P}^{1}$ l'éclaté de $\mathbf{P}^{1} \times \mathbf{P}^{1}$ en $(\infty, \infty) ;$ soit $\mathbf{D}=u^{-1}(\infty, \infty)$ son diviseur exceptionnel. Le groupe de Picard de $\mathrm{S}$ peut être identifié à $\mathbf{Z}^{3}$ de telle sorte que les classes du transformé strict $D^{\prime}$ de $\{\infty\} \times \mathbf{P}^{1}$, du transformé strict $\mathrm{D}^{\prime \prime}$ de $\mathbf{P}^{1} \times\{\infty\}$ et de $\mathrm{D}$ s'identifient respectivement à $(1,0,0),(0,1,0)$ et $(0,0,1)$. L'homomorphisme $u^{*}: \operatorname{Pic}\left(\mathbf{P}^{1} \times \mathbf{P}^{1}\right) \rightarrow \operatorname{Pic}(\mathrm{S})$ s'identifie alors à $(a, b) \mapsto(a, b, a+b)$.

La restriction de $u$ à $u^{-1}\left(\mathbf{A}^{1} \times \mathbf{A}^{1}\right)$ se prolonge en un morphisme $v$ : $\mathrm{S} \rightarrow \mathbf{P}^{2}$. Ce morphisme applique $\mathrm{D}$ sur 1 a droite à l'infini de $\mathbf{P}^{2}$, et $\mathrm{D}^{\prime}$ et $\mathrm{D}^{\prime \prime}$ sur sur les points de $\mathbf{P}^{2}$ de coordonnées homogènes $(1,0,0)$ et $(0,1,0)$ respectivement (dans le système de coordonnées $\left.\left(\underline{z}, \underline{z}^{\prime}, \underline{t}\right)\right)$. Le couple $(\mathrm{S}, v)$ s'identifie à l'éclaté de $\mathbf{P}^{2}$ en ces deux points. L'homomorphisme $v^{*}: \operatorname{Pic}\left(\mathbf{P}^{2}\right) \rightarrow \operatorname{Pic}(\mathrm{S})$ s'identifie à $a \mapsto(a, a, a)$.

Soit Z la transformée stricte de la courbe C par $u$. C'est aussi la transformée stricte de $\widehat{\mathrm{C}}$ par $v$. Notons $m^{\prime}$ et $m^{\prime \prime}$ les multiplicités de $\widehat{\mathrm{C}}$ en $(1,0,0)$ et $(0,1,0)$ respectivement. On a

$$
u^{*}([\mathrm{C}])=[\mathrm{Z}]+m(\mathrm{C})[\mathrm{D}], \quad v^{*}([\widehat{\mathrm{C}}])=[\mathrm{Z}]+m^{\prime}\left[\mathrm{D}^{\prime}\right]+m^{\prime \prime}\left[\mathrm{D}^{\prime \prime}\right],
$$

d'où $\left(d_{\mathrm{C}}, d_{\mathrm{C}}, 2 d_{\mathrm{C}}\right)-m(\mathrm{C})(0,0,1)=(\delta, \delta, \delta)-m^{\prime}(1,0,0)-m^{\prime \prime}(0,1,0)$. il en résulte que l'on a $\delta=2 d_{\mathrm{C}}-m(\mathrm{C})$ et $m^{\prime}=m^{\prime \prime}=d_{\mathrm{C}}-m(\mathrm{C})$.

Remarque. Lorsque C n'est pas réelle, on a $m(\mathrm{C})=0$. Lorsque $\mathrm{C}$ est réelle, $m(\mathrm{C})$ est le nombre de points de $\widetilde{\mathrm{C}}(\mathbf{R})$ dont l'image par l'application composée

$$
\widetilde{\mathrm{C}}(\mathbf{R}) \rightarrow \mathrm{C}(\mathbf{R}) \rightarrow \Gamma(\mathbf{R}) \rightarrow \mathrm{X}(\mathbf{C})=\mathbf{P}^{1}(\mathbf{C})
$$

est le point à l'infini de $\mathbf{P}^{1}(\mathbf{C})$. C'est aussi le nombre de paires de branches infinies de directions asymptotiques opposées de la courbe algébrique réelle affine $(\mathrm{C} \cap \mathrm{U})(\mathbf{R})$. On peut expliciter ce nombre suivant la position du point à l'infini de $\mathbf{P}^{1}(\mathbf{C})$ par rapport à la triangulation canonique de $\mathrm{T}$ :

a) Le point à l'infini de $\mathbf{P}^{1}(\mathbf{C})$ est situé sur une face de la triangulation $\mathrm{T}$

Cela équivaut à dire que $f(\infty)$ n'appartient pas à la droite projective réelle, i.e. que l'on a $\operatorname{deg}(\mathrm{P})=\operatorname{deg}(\mathrm{Q})$ et que le coefficient dominant $\lambda$ de $\mathrm{P}$ n'est pas réel. Dans ce cas le point à l'infini de $\mathbf{P}^{1}(\mathbf{C})$ n'appartient pas à $\Gamma(\mathbf{R})$ et l'on a $m(\mathrm{C})=0$.

b) Le point à l'infini de $\mathbf{P}^{1}(\mathbf{C})$ est situé sur une arête de la triangulation $\mathrm{T}$

Cela équivaut à dire que $f(\infty)$ appartient à la droite projective réelle et est distinct de 0,1 et $\infty$, i.e. que l'on a $\operatorname{deg}(\mathrm{P})=\operatorname{deg}(\mathrm{Q})$ et $\lambda \neq 1$. Dans ce cas le point à l'infini de $\mathbf{P}^{1}(\mathbf{C})$ appartient à une unique arête de $\mathrm{T}$. On a $m(\mathrm{C})=1$ si cette arête est contenue dans $\mathrm{C}(\mathbf{R})$ et $m(\mathrm{C})=0$ sinon. 
c) Le point à l'infini de $\mathbf{P}^{1}(\mathbf{C})$ est un sommet de type 0 (resp. $1 ;$ resp. $\infty$ ) de $\mathrm{T}$

Cela équivaut à dire que $f(\infty)$ est égal à 0 (resp. 1 ; resp. $\infty$ ), i.e. que l'on $\mathrm{a} \operatorname{deg}(\mathrm{P})<\operatorname{deg}(\mathrm{Q})(\operatorname{resp} \cdot \operatorname{deg}(\mathrm{P})=\operatorname{deg}(\mathrm{Q})$ et $\lambda=1 ; \operatorname{resp} \cdot \operatorname{deg}(\mathrm{P})>$ $\operatorname{deg}(\mathrm{Q}))$. Dans ce cas le point à l'infini de $\mathbf{P}^{1}(\mathbf{C})$ correspond à une orbite $\mathrm{A}$ de $\left\langle\sigma_{0}\right\rangle$ (resp. $\left\langle\sigma_{1}\right\rangle$; resp. $\left.\left\langle\sigma_{\infty}\right\rangle\right)$ dans $\mathrm{E}$, et $m(\mathrm{C})$ est le cardinal de $\left\langle\Sigma_{0}\right\rangle \backslash((\mathrm{A} \times \mathrm{A}) \cap \mathcal{O})\left(\operatorname{resp} .\left\langle\Sigma_{1}\right\rangle \backslash((\mathrm{A} \times \mathrm{A}) \cap \mathcal{O}) ;\left\langle\Sigma_{\infty}\right\rangle \backslash\left(\left(\mathrm{A} \times \sigma_{0}(\mathrm{~A})\right) \cap \mathcal{O}\right)\right.$.

7.2.4. Partie homogène de plus haut degré de l'équation affine de $\mathrm{C}$, lorsque $\mathrm{X}=\mathbf{P}^{1}$.

Conservons les notations des numéros 7.2 .2 et 7.2 .3 . Il résulte de la prop. 7.2 que l'équation de la courbe algébrique affine $\mathrm{C} \cap\left(\mathrm{A}^{1} \times \mathrm{A}^{1}\right)$ (dans chacun des systèmes de coordonnées $(x, y)$ et $\left(z, z^{\prime}\right)$ ) est de degré $2 d_{\mathrm{C}}-m(\mathrm{C})$. Si $\mathrm{C}$ est réelle, on choisit pour chaque paire de branches infinies de $(\mathrm{C} \cap \mathrm{U})(\mathbf{R})$, de directions asymptotiques opposées, une forme linéaire $\ell_{j}$ en $x, y$ qui est une équation de la droite vectorielle parallèle à ces directions.

Proposition 7.3. La partie homogène de plus haut degré de l'équation de $\mathrm{C} \cap\left(\mathrm{A}^{1} \times \mathrm{A}^{1}\right)$ dans le système de coordonnées $(x, y)$ est un multiple scalaire de

$$
\left(x^{2}+y^{2}\right)^{d_{\mathrm{C}}-m(\mathrm{C})} \prod_{j=1}^{m(\mathrm{C})} \ell_{j} .
$$

Au cours de la démonstration de la prop. 7.2, nous avons vu que la courbe $\widehat{\mathrm{C}}$ a pour multiplicité $d_{\mathrm{C}}-m(\mathrm{C})$ en les points de coordonnées homogènes $(1,0,0)$ et $(0,1,0)$ dans le système de coordonnées $\left(\underline{z}\right.$, underline $\left.z^{\prime}, \underline{t}\right)$, i.e. $(1, i, 0)$ et $(1,-i, 0)$ dans le système de coordonnées $(\underline{x}, \underline{y}, \underline{t})$. Il en résulte que la partie homogène de plus haut degré de l'équation de $\mathrm{C} \cap\left(\mathrm{A}^{1} \times \mathrm{A}^{1}\right)$ est multiple de $\left(x^{2}+y^{2}\right)^{d_{\mathrm{C}}-m(\mathrm{C})}$. Elle est aussi multiple de chacune des formes linéaires $\ell_{i}$. Comme son degré est $2 d_{\mathrm{C}}-m(\mathrm{C})$, la prop. $7.3 \mathrm{~s}$ 'en suit.

Remarque. Il résulte de la prop. 7.3 que, hormis en les deux points $(1, i, 0)$ et $(1,-i, 0)$ (dans le système de coordonnées $(\underline{x}, \underline{y}, \underline{t})$ ), la droite à l'infini de $\mathbf{P}^{2}$ ne coupe $\widetilde{\mathrm{C}}$ qu'en des points lisses et transversalement. Il en résulte que chaque branche infinie de $\mathrm{C}(\mathbf{R})$ est asymptote à une droite (qui est la même pour des branches infinies de directions asymptotiques opposées).

Exemples. 1) Reprenons le dessin combinatoire du $\S 3.8$, défini par $\mathrm{E}=$ $\{a, b, c, d, e\}, \sigma_{0}=(a)(b c)(d e)$ et $\sigma_{1}=(a b)(c d)(e)$.

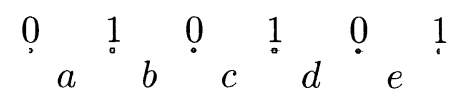


L'ensemble $\mathrm{E} \times \mathrm{E}$ possède trois orbites sous l'action de $\left\langle\Sigma_{0}, \Sigma_{1}\right\rangle$ :

$$
\begin{aligned}
\mathcal{O}_{1} & =\{a a, b b, c c, d d, e e\}, \\
\mathcal{O}_{2} & =\{a b, a c, b d, c e, d e, e d, e c, d b, c a, b a\}
\end{aligned}
$$

et

$$
\mathcal{O}_{3}=\{a d, a e, b e, c d, d c, e b, e a, d a, c b, b c\}
$$

Les multiplicités de composantes irréductibles de $\Gamma_{(\mathbf{C})}$ en $(\infty, \infty)$ dépendent du système de coordonnées choisi. Ce dessin est totalement ramifié au-dessus de l'infini et on peut imposer à l'unique élément de la fibre audessus de l'infini d'être en l'infini. Auquel cas, compter les multiplicités en $(\infty, \infty)$ de chaque composante revient à compter le cardinal de $\left\langle\Sigma_{\infty}\right\rangle \backslash \mathcal{O}_{i}$ $(1 \leq i \leq 3)$. Comme

$$
\begin{gathered}
\Sigma_{\infty}=(a a, c c, e e, d d, b b)(a c, c e, e d, d b, b a)(a e, c d, e b, d a, b c) \\
(a d, c b, e a, d c, b e)(a b, c a, e c, d e, b d),
\end{gathered}
$$

on a $m\left(\mathrm{C}_{1}\right)=1, m\left(\mathrm{C}_{2}\right)=2$, et $m\left(\mathrm{C}_{3}\right)=2$. Par ailleurs $d_{\mathrm{C}_{1}}=1, d_{\mathrm{C}_{2}}=2$, $d_{\mathrm{C}_{3}}=2$ (chacun étant égal au cardinal de $\mathrm{O}_{i}$ divisé par 5 ). Il en résulte que les degrés de ces composantes sont respectivement 1, 2 et 2.

On retrouve ces résultats par un calcul direct. En effet, une fonction de Belyi associée à $\left(\mathrm{E}, \sigma_{0}, \sigma_{1}\right)$ est le polynôme $f(z)=8 z^{5}-10 z^{3}+\frac{5}{2} z+\frac{1}{2}$. On a

$$
f(z)=\frac{1}{2}(z+1)\left(4 z^{2}-2 z-1\right)^{2}=8 \prod_{n=0}^{4}\left(z+\cos \frac{2 \pi n}{5}\right) .
$$

Cela signifie que $\mathbf{P}^{1}(\mathbf{C})$ muni du graphe bicolorié $f^{-1}([0,1])$ est isomorphe au dessin de départ. (La fonction de Belyi précédente a été normalisée de telle sorte que le sommet gauche du dessin, le sommet droit du dessin et le point à l'infini correspondent respectivement aux points $-1,1$ et $\infty$ de $\mathbf{P}^{1}(\mathbf{C})$.)

L'image réciproque de $\mathbf{P}^{1}(\mathbf{R})$ par $f$ est une courbe correspondant à la figure $3 \mathrm{du}$ §3.8. Elle a trois composantes irréductibles : une droite horizontale et deux hyperboles. Cela se voit en décomposant $\operatorname{Im} f(x+i y)$ en facteurs irréductibles : on trouve

$\operatorname{Im} f(x+i y)=40 y\left(x^{2}-\frac{5+2 \sqrt{5}}{5} y^{2}-\frac{3+\sqrt{5}}{8}\right)\left(x^{2}-\frac{5-2 \sqrt{5}}{5} y^{2}-\frac{3-\sqrt{5}}{8}\right)$.

Les degrés des facteurs irréductibles sont bien égaux à 1,2 et 2 .

Enfin, les parties homogènes de plus haut degré de ces composantes sont respectivement

$$
\begin{array}{ll}
y & \text { pour } \mathcal{O}_{1}, \\
(\sin (\pi / 5) x-\cos (\pi / 5) y)(\sin (4 \pi / 5) x-\cos (4 \pi / 5) y) & \text { pour } \mathcal{O}_{2}, \\
(\sin (2 \pi / 5) x-\cos (2 \pi / 5) y)(\sin (3 \pi / 5) x-\cos (3 \pi / 5) y) & \text { pour } \mathcal{O}_{3} .
\end{array}
$$


2) Reprenons le dessin combinatoire et les notations du $§ 6.1$

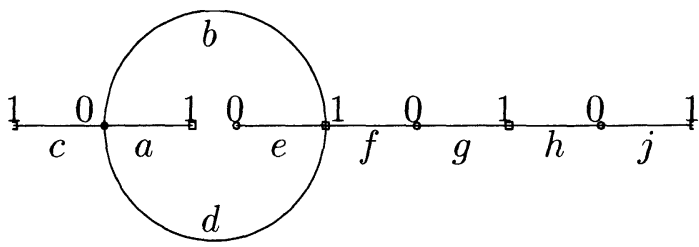

On a respectivement $d_{\mathrm{C}_{1}}=1, d_{\mathrm{C}_{2}}=6, d_{\mathrm{C}_{3}}=2$. Les multiplicités en $(\infty, \infty)$ de chaque composante dépendent du système de coordonnées choisi. Si on décide que le point $\mathrm{P}$ au-dessus de l'infini d'indice de ramification 6 se trouve à l'infini, alors la multiplicité en $(\infty, \infty)$ d'une composante $\mathrm{C}_{i}$ est égale au nombre d'éléments de $\left\langle\Sigma_{\infty}\right\rangle \backslash \mathcal{O}_{i}$ dont l'image dans $\left\langle\sigma_{\infty}\right\rangle \backslash \mathrm{E} \times$ $\left\langle\sigma_{\infty}^{*}\right\rangle \backslash \mathrm{E}$ correspond au point $\mathrm{P}$. Ainsi, on a $m\left(\mathrm{C}_{1}\right)=1, m\left(\mathrm{C}_{2}\right)=4$ et $m\left(\mathrm{C}_{3}\right)=1$. On en déduit que les degrés des courbes représentées sont respectivement 1,8 et 3 .

On en déduit entre autre que les parties homogènes de plus haut degré sont respectivement

$$
\begin{array}{ll}
y & \text { pour } \mathcal{O}_{1} \\
\left(x^{2}+y^{2}\right)^{2}\left(x^{2}-3 y^{2}\right)\left(3 x^{2}-y^{2}\right) / 16 & \text { pour } \mathcal{O}_{2} \\
\left(x^{2}+y^{2}\right) x & \text { pour } \mathcal{O}_{3}
\end{array}
$$

\subsection{Le cas où $X$ est connexe de genre quelconque.}

Dans ce numéro, nous supposons que $\mathrm{X}$ est connexe, de genre quelconque. On note $\operatorname{Jac}(\mathrm{X})$ la jacobienne de $\mathrm{X}$ et $\mathrm{Jac}\left(\mathrm{X}^{*}\right)$ celle de $\mathrm{X}^{*}$. Rappelons (cf. §7.1.6) que l'on dispose d'une suite exacte canonique

(1) $0 \rightarrow \operatorname{Pic}(\mathrm{X}) \times \operatorname{Pic}\left(\mathrm{X}^{*}\right) \rightarrow \operatorname{Pic}\left(\mathrm{X} \times \mathrm{X}^{*}\right) \rightarrow \operatorname{Hom}\left(\operatorname{Jac}(\mathrm{X}), \operatorname{Jac}\left(\mathrm{X}^{*}\right)\right) \rightarrow 0$,

Toute composante irréductible $\mathrm{C}$ de $\Gamma_{(\mathbf{C})}$ est un diviseur de $\mathrm{X} \times \mathrm{X}^{*}$. Nous notons $[\mathrm{C}]$ sa classe dans $\operatorname{Pic}\left(\mathrm{X} \times \mathrm{X}^{*}\right)$ et $u_{\mathrm{C}}$ l'image de $[\mathrm{C}]$ dans le groupe $\operatorname{Hom}\left(\operatorname{Jac}(\mathrm{X}), \operatorname{Jac}\left(\mathrm{X}^{*}\right)\right)$.

7.3.1. La somme des $u_{\mathrm{C}}$ est nulle.

Considérons sur $\mathrm{X} \times \mathrm{X}^{*}$ la fonction rationnelle $\varphi=f \circ p_{1}-f^{*} \circ p_{2}$, où $p_{1}$ et $p_{2}$ sont les projections sur $\mathrm{X}$ et $\mathrm{X}^{*}$. Le diviseur de $\varphi$ est

$$
\operatorname{div}(\varphi)=\sum_{\mathrm{C}} \mathrm{C}-\sum_{x \in f^{-1}(\infty)} e_{x, f}\{x\} \times \mathrm{X}^{*}-\sum_{y \in\left(f^{*}\right)^{-1}(\infty)} e_{y, f^{*}} \mathrm{X} \times\{y\},
$$

où $e_{x, f}$ et $e_{y, f^{*}}$ désignent les indices de ramification de $f$ et $f^{*}$ en $x$ et $y$ respectivement. Il en résulte que l'élément $\sum_{\mathrm{C}}[\mathrm{C}]$ de $\mathrm{Pic}\left(\mathrm{X} \times \mathrm{X}^{*}\right)$ appartient à l'image de $\operatorname{Pic}(\mathrm{X}) \times \operatorname{Pic}\left(\mathrm{X}^{*}\right)$ par la première flèche de $(1)$, donc que l'on a

$$
\sum_{\mathrm{C}} u_{\mathrm{C}}=0 \text {. }
$$


7.3.2. Certains des $u_{\mathrm{C}}$ peuvent être non nuls.

Nous allons dans ce numéro montrer par un exemple que, bien que la somme des $u_{\mathrm{C}}$ soit nulle, certains des $u_{\mathrm{C}}$ peuvent être non nuls.

Prenons pour X la courbe elliptique d'équation homogène $y^{2} t=x^{3}+t^{3}$ dans $\mathbf{P}^{2}$ et pour $f$ le morphisme $(x, y, t) \mapsto(y+t, 2 t)$ de $\mathbf{X}$ dans $\mathbf{P}^{1}$. Alors $(\mathrm{X}, f)$ est un revêtement ramifié de degré 3 de $\mathbf{P}^{1}$, non ramifié audessus de $\mathbf{P}^{1}-\{0,1, \infty\}$. L'ensemble $f^{-1}(0)$ (resp. $f^{-1}(1)$; resp. $f^{-1}(\infty)$ ) possède pour seul point $(0,-1,1)$ (resp. $(0,1,1)$; resp. $(0,1,0))$, et l'indice de ramification de $f$ en ce point est 3 .

Le dessin combinatoire associé à ce revêtement est isomorphe à $\left(\mathrm{E}, \sigma_{0}, \sigma_{1}\right)$ où $\mathrm{E}=\{a, b, c\}$ et $\sigma_{0}=\sigma_{1}=(a b c)$. Le dessin d'enfant correspondant peut être représenté comme suit :

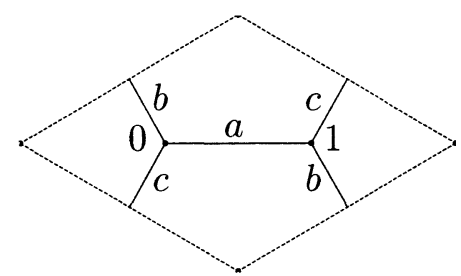

(étant entendu que la surface sur laquelle il est tracé, qui est de genre 1, est le tore obtenu en identifiant deux à deux les côtés opposés du losange représentés en pointillés). Le dessin d'enfant triangulé correspondant peut être représenté avec les mêmes conventions comme suit :

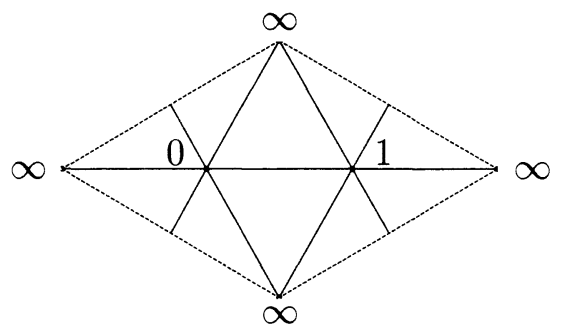

Pour l'action du groupe $\left\langle\Sigma_{0}, \Sigma_{1}\right\rangle, \mathrm{E} \times \mathrm{E}$ possède trois orbites $\mathcal{O}_{1}, \mathcal{O}_{2}, \mathcal{O}_{3}$ sur lesquelles $\Sigma_{0}$ et $\Sigma_{1}$ opèrent comme suit (en écrivant $x y$ l'élément $(x, y)$ de $\mathrm{E} \times \mathrm{E}$ pour alléger les notations)

$$
\begin{aligned}
& \Sigma_{0}\left|\mathcal{O}_{1}=\Sigma_{1}\right| \mathcal{O}_{1}=(a a, b c, c b), \\
& \Sigma_{0}\left|\mathcal{O}_{2}=\Sigma_{1}\right| \mathcal{O}_{2}=(b b, c a, a c), \\
& \Sigma_{0}\left|\mathcal{O}_{3}=\Sigma_{1}\right| \mathcal{O}_{3}=(c c, a b, b a) .
\end{aligned}
$$

Ces orbites sont stables par l'involution $x y \mapsto y x$. Elles correspondent donc à trois composantes irréductibles $\mathrm{C}_{1}, \mathrm{C}_{2}, \mathrm{C}_{3}$ de $\Gamma_{(\mathbf{C})}$, toutes réelles.

Comme la courbe $\mathrm{X}$ est définie sur $\mathbf{R}$, elle est canoniquement isomorphe à $\mathrm{X}^{*}$ et $\Gamma_{(\mathbf{C})}$ s'identifie alors à l'image réciproque de la diagonale de $\mathbf{P}^{1} \times \mathbf{P}^{1}$ par le morphisme $f \times f: \mathrm{X} \times \mathrm{X} \rightarrow \mathbf{P}^{1} \times \mathbf{P}^{1}$. Ces identifications étant faites, 
$\mathrm{C}_{1}, \mathrm{C}_{2}, \mathrm{C}_{3}$ s'identifient respectivement aux graphes de $\mathrm{Id}_{\mathrm{X}}, u$ et $u^{2}$, où $u$ est l'automorphisme $(x, y, t) \mapsto(j x, y, t)$ de $\mathrm{X}$, avec $j=\frac{-1+i \sqrt{3}}{2}$.

Comme $\mathrm{X}$ est de genre 1 , nous pouvons identifier la jacobienne $\mathrm{Jac}(\mathrm{X})$ à $\mathrm{X}$, l'élément neutre de $\mathrm{Jac}(\mathrm{X})$ correspondant au point à l'infini $(0,1,0)$ de $\mathrm{X}$. Identifions de même $\mathrm{Jac}\left(\mathrm{X}^{*}\right)$ à $\mathrm{X}^{*}$, donc à $\mathrm{X}$. Il résulte alors de l'alinéa précédent que les morphismes $u_{\mathrm{C}_{1}}, u_{\mathrm{C}_{2}}, u_{\mathrm{C}_{3}}$ de $\operatorname{Jac}(\mathrm{X})$ dans $\operatorname{Jac}\left(\mathrm{X}^{*}\right)$ s'identifient à $\operatorname{Idx}_{\mathrm{X}}, u$ et $u^{2}$. En particulier, ils sont tous trois non nuls.

\section{Bibliographie}

[1] Сh. Birkenhake. H. Lange, Complex Abelian Varieties. Grundlehren der mathematischen Wissenchaften 302, A Series of Comprehensive Studies in Mathematics, Springer-Verlag, 1992.

[2] L. Pharamond dit D'Costa, Géométrie réelle des dessins d'enfant. Journal de théorie des nombres de Bordeaux 16 (2004), 639-691.

Layla Pharamond dit D'Costa

Lyçée Chaptal

6 allée Chaptal

22000 Saint-Brieuc

E-mail : pharamond@math.jussieu.fr 